\title{
Kinetic theory of spin-polarized systems in electric and magnetic fields with spin-orbit coupling: II. RPA response functions and collective modes
}

\author{
K. Morawetz ${ }^{1,2,3}$ \\ ${ }^{1}$ Münster University of Applied Sciences, Stegerwaldstrasse 39, 48565 Steinfurt, Germany \\ ${ }^{2}$ International Institute of Physics (IIP) Federal University of Rio Grande \\ do Norte Av. Odilon Gomes de Lima 1722, 59078-400 Natal, Brazil and \\ 3 Max-Planck-Institute for the Physics of Complex Systems, 01187 Dresden, Germany
}

\begin{abstract}
The spin and density response functions in the random phase approximation (RPA) are derived by linearizing the kinetic equation including a magnetic field, the spin-orbit coupling, and mean fields with respect to an external electric field. Different polarization functions appear describing various precession motions showing Rabi satellites due to an effective Zeeman field. The latter turns out to consist of the mean-field magnetization, the magnetic field, and the spin-orbit vector. The collective modes for charged and neutral systems are derived and a threefold splitting of the spin waves dependent on the polarization and spin-orbit coupling is shown. The dielectric function including spin-orbit coupling, polarization and magnetic fields is presented analytically for long wave lengths and in the static limit. The dynamical screening length as well as the long-wavelength dielectric function shows an instability in charge modes, which are interpreted as spin segregation and domain formation. The spin response describes a crossover from damped oscillatory behavior to exponentially damped behavior dependent on the polarization and collision frequency. The magnetic field causes ellipsoidal trajectories of the spin response to an external electric field and the spin-orbit coupling causes a rotation of the spin axes. The spin-dephasing times are extracted and discussed in dependence on the polarization, magnetic field, spin-orbit coupling and single-particle relaxation times.

PACS numbers: $\quad$ 75.30.Fv, 71.70.Ej, 85.75.Ss, 77.22.Ch
\end{abstract}

\section{INTRODUCTION}

The development of spintronic devices is largely based on the understanding of collective spin waves. Spin waves, besides density waves, are one of the fundamental collective excitations in strongly interacting Fermi systems, e.g., in ferromagnetic materials ${ }^{112}$, graphene ${ }^{314}$, or isospin excitations in nuclear matter ${ }^{5}$. In the past, this had motivated people to develop Green functions techniques for the quantum transport theory of spin resonance $e^{6 / 7}$.

If the range of interaction is shorter than the DeBroglie wavelength, such excitations are also predicted ${ }^{8} 13$ and observed ${ }^{14115}$ in dilute spin-polarized gases. The transverse spin-wave dynamics has been the subject of a series of theoretical investigations $\frac{12116}{}$. In ultracold gases, spin waves have been observed, even spatially resolved $17 / 18$, and were described by longitudinal spin waves $\frac{19}{}$. The spin diffusion in trapped Bose gases shows an anisotropy in modes ${ }^{20}$ and the collisionless damping has been seen to deviate for quadrupole modes from experiments 21 indicating the role of collisional correlations. The spin-wave damping has been measured in a polarized Fermi-liquidlike ${ }^{3} \mathrm{He}-{ }^{4} \mathrm{He}$ mixture even at zero temperature ${ }^{22}$ and as an 'identical spin-rotation effect 23 .

The influence of the magnetic field on such spin waves is of special interest, e.g., as magneto-transport effects in paramagnetic gases 24 . The Landau levels influence the spin relaxation $25 \mid 26$, which has been measured with the help of spin coherence times 27 . The influence of magnetic fields is treated in various systems ranging from plasma $2^{28}$, solid-state plasmas ${ }^{29}$, and semiconductor ${ }^{30}$ to spin-orbit coupled systems ${ }^{31}$ and graphene ${ }^{32}$. The feedback of magnetization dynamics due to spins on the spin dynamics itself is reviewed in Ref ${ }^{[3}$. The Zeeman field is reported to trigger a transition from a charge density wave to a spin density wave ${ }^{34}$. Quite promising for technological applications turns out the possibility to create magnetic nanooscillations by pure spin currents 35 . The spin current can be converted into a terahertz electromagnetic pulse due to the inverse spin Hall effect ${ }^{36}$.

Quite recently the spin-orbit coupling has moved to the center of interest $\frac{37 / 38}{}$ since this coupling allows to convert spin waves into spin currents, which is important for spintronic devices 39 . There has been observed a spin-orbit-driven ferromagnetic resonance ${ }^{40}$ which shows that an effective magnetic field is created in the magnetic material by oscillating electric currents. This is also the

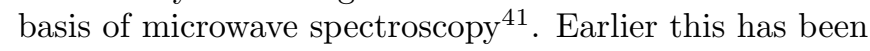
identified as a magneto-electric effect where a charge current induces a spin polarization known as the Edelstein effect 42143 . Spin-polarized longitudinal currents can be induced due to spin-orbit interaction in certain crystal symmetries ${ }^{44}$. Experimentally even a planar Hall effect has been reported using spin waves ${ }^{45}$ as well as spin polarization oscillations without spin precession 46 .

Coulomb interactions are known to reduce the effect of spin-orbit coupling in the spin-Hall effect ${ }^{47}$. The phononmodulated spin-orbit interaction has been investigated to show that the screening is influenced by the spin-orbit coupling 4 . Screening effects play a crucial role for the temperature dependence of conductivity in quasi two- 
dimensional systems ${ }^{49}$, monolayer graphene ${ }^{[50}$ and multilayer graphene $e^{51}$. The Coulomb correction to the conductivity in graphene had covered an involved debate 52 55. With this respect the extraction of correct spin relaxation or dephasing times has been in the center of interest $\frac{56 \mid 58}{58}$ since it is most promising for new storage devices.

During the last few years, many researchers have shown an appreciable interest in the dielectric function and the properties of screening in two-dimensional gases with spin-orbit coupling 69 . Similar results appear if the pseudospin response function in doped graphene is calculated 62 65. The random phase approximation (RPA) is calculated in this respect for single and multilayer graphene 66167. These approaches calculate the Lindhard dielectric function with form factors arising from chirality subbands. Additional energy denominators appear if four-band approximations are considered ${ }^{65}$ where a band gap appears 68 . The comparison of pristine graphene, Dirac cones, and gaped graphene with an antidot lattice can be found in Ref. $\frac{69}{}$. These responses are needed if one wants to understand the optical properties of graphene irradiated by an external electric field ${ }^{70}$.

All of these approaches consider the spin degree of freedom as an inner property of particles leading to form factors in the Lindhard dielectric function. Here, the spin degree of freedom is considered on equal statistical footing with the particle distribution leading to more forms of the response function due to the spin-orbit coupling, satellites, and Zeeman splitting by magnetic fields and self-energy effects that cannot be cast into a Lindhard form with form factors. This has an impact on the collective density and spin modes. The will calculate analytically the threefold splitting of spin modes ${ }^{71}$ as a function of the spin-orbit coupling and the effective Zeeman field.

In this paper, we want to present a unifying treatment of density and spin waves in the random phase approximation including the spin-orbit coupling, magnetic fields, and an arbitrary magnetization for systems with charged and neutral scattering. This will allow us to investigate the influence of spin-orbit coupling on the screening properties of the Coulomb interaction as well as the collective modes in systems with neutral scattering. For this purpose, we linearize the kinetic equation derived in the first part of this paper. Linearizing the mean-field kinetic equation yields the RPA response since a lowerlevel kinetic equation provides a response of higher-order many-body correlations ${ }^{72}$.

Following a short summary of the basic kinetic equation derived in the first part of the paper, the linear response to an external electric field is presented in the second section. This results into coupled equations for the spin and density response with a variety of dynamical polarization functions describing different precessions. In Sec. III, the charge and spin density response functions are analyzed with respect to their collective modes and the spin waves are discussed for neutral and charged scattering. The polarization causes a splitting of spin modes. For certain polarizations and spin-orbit coupling, an in- stability occurs, which is interpreted as spin-domain separation. This is underlined by the influence of spin-orbit coupling and polarization on the screening properties in charged systems where the instability occurs in spatial domain. The dielectric function including the spinorbit coupling and an effective medium-dependent Zeeman field is derived analytically in the long-wavelength and static limits respectively. The dynamic and static screening lengths are discussed there. The spin response due to an applied electric field is then extracted and the spin-dephasing times are discussed. As in the Edelstein effect, the applied electric field causes a charge current and a spin response that shows oscillations dependent on the spin-orbit coupling, magnetic field, and relaxation time. In Sec. V, we present the linear response including arbitrary magnetic fields and show how the normal Hall and quantum Hall effects appear from the kinetic theory. As an important point, a subtlety in retardations due to the magnetic field is presented. A summary finishes this second part of the paper. In Appendix, some useful expressions for solving involved vector equations are presented.

Let us now shortly summarize the quantum kinetic equation derived in the first part of the paper. We describe the density and polarization density by their corresponding Wigner functions

$$
\sum_{p} f(\vec{x}, \vec{p}, t)=n(\vec{x}, t), \quad \sum_{p} \vec{g}(\vec{x}, \vec{p}, t)=\vec{s}(\vec{x}, t)
$$

where $\sum_{p}=\int d^{D} p /(2 \pi \hbar)^{D}$ for $D$ dimensions. As a result of the first part of this paper, the four Wigner functions

$$
\hat{\rho}=f+\vec{\sigma} \cdot \vec{g}=\left(\begin{array}{cc}
f+g_{z} & g_{x}-i g_{y} \\
g_{x}+i g_{y} & f-g_{z}
\end{array}\right)
$$

have been shown to obey coupled kinetic equations

$$
\begin{aligned}
D_{t} f+\vec{A} \cdot \vec{g} & =0 \\
D_{t} \vec{g}+\vec{A} f & =2(\vec{\Sigma} \times \vec{g})
\end{aligned}
$$

where $D_{t}=\left(\partial_{t}+\overrightarrow{\mathcal{F}} \overrightarrow{\partial_{p}}+\vec{v} \overrightarrow{\partial_{x}}\right)$ describes the drift and force of the scalar and vector part with the velocity

$$
v=\frac{p}{m_{e}}+\partial_{p} \Sigma_{0}
$$

and the effective Lorentz force

$$
\overrightarrow{\mathcal{F}}=\left(e \vec{E}+e \vec{v} \times \vec{B}-\overrightarrow{\partial_{x}} \Sigma_{0}\right) .
$$

This effective Lorentz force as well as the velocity both become modified due to the scalar meanfield selfenergy

$$
\Sigma_{0}(\vec{q}, t)=n(\vec{q}, t) V_{0}(\vec{q})+\vec{s}(\vec{q}, t) \cdot \vec{V}(\vec{q})
$$

as a spatial convolution between the density and spin polarization with the Fourier transformed scalar and vector potentials, respectively. The latter ones originate from 
magnetic impurities and/or effective magnetizations in the material. Here, we concentrate on the intrinsic spinorbit coupling. The meanfields with extrinsic spin-orbit coupling are given in III.C of the first part of the paper.

The second parts on the left side of (3) represent the coupling between the spin parts of the Wigner distribution given by the vector drift

$$
A_{i}=\left(\vec{\partial}_{p} \Sigma_{i} \vec{\partial}_{x}-\vec{\partial}_{x} \Sigma_{i} \vec{\partial}_{p}+e\left(\vec{\partial}_{p} \Sigma_{i} \times \vec{B}\right) \vec{\partial}_{p}\right) .
$$

We subsumed in the vector selfenergy

$$
\vec{\Sigma}=\vec{\Sigma}_{H}(\vec{x}, t)+\vec{b}(\vec{x}, \vec{p}, t)+\mu_{B} \vec{B}(\vec{x}, t)
$$

the magnetic impurity meanfield

$$
\vec{\Sigma}_{H}=n(\vec{q}, t) \vec{V}(q)+\vec{s}(\vec{q}, t) V_{0}(q)
$$

and the spin-orbit coupling vector $\vec{b}$, as well as the Zeeman term $\mu_{B} \vec{B}$ such that the effective Hamiltonian possesses the Pauli structure

$$
H_{\mathrm{eff}}=H+\vec{\sigma} \cdot \vec{\Sigma}
$$

with the effective scalar Hamiltonian

$$
H=\frac{k^{2}}{2 m_{e}}+\Sigma_{0}(\vec{x}, \vec{k}, T)+e \Phi(\vec{x}, T)
$$

where $\vec{k}=\vec{p}-e \vec{A}(\vec{x}, t)$ ensures gauge invariance. Any spin-orbit coupling found in the literature can be recast into the form $\vec{\sigma} \cdot \vec{b}(p)$ as illustrated in Table I of the first part of this paper. The vector part of (3) finally contains additionally the spin-rotation term on the right-hand side responsible for spin precession.

\section{LINEAR RESPONSE}

\section{A. Without magnetic field but conserving relaxation time}

Let us consider the linearization of the kinetic equation (3) with respect to an external electric field, no magnetic field and in a homogeneous situation. We Fourier transform the time $\partial_{t} \rightarrow-i \omega$ and the spatial coordinates $\vec{\partial}_{x} \rightarrow i \vec{q}$. The Wigner functions are linearized according to $\hat{\rho}(\vec{x}, \vec{p}, t)=f(\vec{p})+\delta f(\vec{x}, \vec{p}, t)+\vec{\sigma} \cdot[\vec{g}(\vec{p})+\delta \vec{g}(\vec{x}, \vec{p}, t)]$ due to the external electric field perturbation $e \delta \vec{E}=$ $e \vec{E}(\vec{x}, t)=-\nabla \Phi$. The density and spin-density variation reads

$$
\begin{aligned}
& \delta n(\vec{q}, \omega)=\sum_{p} \delta f(\vec{q}, \vec{p}, \omega) \\
& \delta \vec{s}(\vec{q}, \omega)=\sum_{p} \delta \vec{g}(\vec{q}, \vec{p}, \omega)
\end{aligned}
$$

and the density and spin-density linear response functions are given by

$$
\begin{aligned}
\delta n(\vec{q}, \omega) & =\chi(\vec{q}, \omega) \Phi \\
\delta \vec{s}(\vec{q}, \omega) & =\vec{\chi}^{s}(\vec{q}, \omega) \Phi .
\end{aligned}
$$

Further, we assume a collision integral of the relaxation time approximation 73

$$
-\frac{1}{2}\left[\hat{\tau}^{-1}, \delta \hat{\rho}^{l}\right]_{+}
$$

with the vector and scalar parts of the relaxation times $\hat{\tau}^{-1}=\tau^{-1}+\vec{\sigma} \cdot \vec{\tau}^{-1}$ where

$$
\tau=\frac{\tau^{-1}}{\tau^{-2}-\left|\vec{\tau}^{-1}\right|^{2}}, \quad \vec{\tau}=-\frac{\vec{\tau}^{-1}}{\tau^{-2}-\left|\vec{\tau}^{-1}\right|^{2}} .
$$

In the first part of the paper, the relaxation of the kinetic equations (3) has been shown towards the twoband distribution $f=\frac{f_{+}+f_{-}}{2}$ and $\vec{g}=\vec{e} \frac{f_{+}-f_{-}}{2}$ with the Fermi-Dirac distribution

$$
f_{0}\left(\epsilon_{p}(r) \pm|\vec{\Sigma}(p, r)|\right)
$$

and the precession direction $\vec{e}=\vec{\Sigma} / \Sigma$. Now we assume a relaxation towards a local distribution $f^{l}=$ $f_{0}(\epsilon \pm|\Sigma|-\mu-\delta \mu)$ such that the density conservation can be enforced ${ }^{7475}$,

$$
\begin{aligned}
\delta n & =\sum_{p}\left(f-f_{0}\right)=\sum_{p}\left(f-f^{l}+f^{l}-f_{0}\right) \\
& =\sum_{p}\left(f^{l}-f_{0}\right)=\partial_{\mu} n \delta \mu,
\end{aligned}
$$

as expressed by the second line. Therefore the scalar relaxation term becomes

$$
-\frac{\delta \hat{\rho}^{l}}{\tau}=-\frac{\delta \hat{\rho}}{\tau}+\frac{\delta n}{\tau \partial_{\mu} n} \partial_{\mu} \hat{\rho}_{0} .
$$

In this way the density is conserved in the response function which could be extended to include more conservation laws ${ }^{76 / 77}$. If we consider only the density conservation but not the polarization conservation, we can restrict ourselves to the $\partial_{\mu} f_{0}$ term. Please note that we neglect in this way the interference effects of disorder 78 .

Abbreviating now $-i \omega+i \vec{p} \cdot \vec{q} / m+\tau^{-1}=a$ and $i q \partial_{p} \vec{\Sigma}+$ $\vec{\tau}^{-1}=\overrightarrow{\mathcal{B}}$, the coupled kinetic equations 3 take then the form

$$
\begin{aligned}
a \delta f+\overrightarrow{\mathcal{B}} \delta \vec{g} & =S_{0} \\
a \delta \vec{g}+\overrightarrow{\mathcal{B}} \delta f-2 \vec{\Sigma} \times \delta \vec{g} & =\vec{S}
\end{aligned}
$$

with $e \vec{E}=-i \vec{q} \Phi$ and

$$
\begin{aligned}
S_{0} & =i q \partial_{p} f\left(\Phi+\delta \Sigma_{0}\right)+i q \partial_{p} \vec{g} \cdot \delta \vec{\Sigma}+\frac{\delta n}{\tau \partial_{\mu} n} \partial_{\mu} f_{0} \\
\vec{S} & =i q \partial_{p} \vec{g}\left(\Phi+\delta \Sigma_{0}\right)+i q \partial_{p} f \delta \vec{\Sigma}+2(\delta \vec{\Sigma} \times \vec{g})+\frac{\delta n \partial_{\mu} \vec{g}}{\tau \partial_{\mu} n} .
\end{aligned}
$$

In order to facilitate the vector notation we want to understand $q \partial_{p}=\vec{q} \cdot \overrightarrow{\partial_{p}}$ in the following. 


\section{B. Collective modes from balance equations}

Multiplying the linearized kinetic equations with powers of momentum and integrating one obtains coupled hierarchies of moments. A large variety of treatments neglect certain Landau-liquid parameters ${ }^{79}$ based on the work of Ref ${ }^{80}$ in order to close such system. A more advanced closing procedure was provided by Ref. where the energy dependence of $\delta \vec{s}$ was assumed to be factorized from space and direction $\vec{p}$ dependencies.

We will not follow these approximations here but solve the linearized equation exactly to provide the solution of the balance equations and the dispersion. Amazingly, this yields a quite involved and extensive structure with much more terms than usually presented in the literature. Nevertheless, it is instructive to have a first look at the balance equation for the densities

$$
\begin{gathered}
\partial_{t} \delta n+\partial_{x_{i}} \vec{J}_{i}+\vec{\tau}^{-1} \cdot \delta \vec{s}=0 \\
\partial_{t} \delta \vec{s}+\partial_{x_{i}} \vec{S}_{i}+\vec{\tau}^{-1} \delta n-2 \sum_{p} \vec{\Sigma} \times \delta \vec{g}=2 \delta \vec{\Sigma} \times \vec{s}
\end{gathered}
$$

where we Fourier transformed the wavevector $q$ back to spatial coordinates $x$. Then the density currents and magnetization currents

$$
\begin{aligned}
\hat{J}_{j}=\frac{1}{2} \sum_{p}\left[\hat{\rho}, v_{j}\right]_{+} & =\sum_{p}\left[f v_{j}+\vec{g} \cdot \partial_{p_{j}} \vec{b}+\vec{\sigma} \cdot\left(v_{j} \vec{g}+f \partial_{p_{j}} \vec{b}\right)\right] \\
& =J_{j}+\vec{\sigma} \cdot \vec{S}_{j} .
\end{aligned}
$$

appear exactly as expected from the elementary definitions, see Sec. III.G of part I.

We are now interested in the long wavelength limit $q \rightarrow 0$, which means we neglect any spatial derivative in (21). Alternatively, we might consider this as the spatially integrated values providing the change of number of particles and magnetization

$$
N=\int d^{3} x n(x)=n_{q=0}, \quad \vec{m}=\int d^{3} x \vec{s}(x)=\vec{s}_{q=0}
$$

The first equation of 21 gives in frequency space

$$
-i \omega \delta N+\vec{\tau}^{-1} \cdot \delta \vec{m}=0
$$

with the help of which we get the closed equation for the magnetization from 21

$$
\begin{aligned}
& -i \omega \delta \vec{m}-\frac{i}{\omega}\left(\vec{\tau}^{-1}+2 \vec{m} \times \vec{V}\right) \vec{\tau}^{-1} \cdot \delta \vec{m}-2\left(N \vec{V}+\mu_{B} \vec{B}\right) \times \delta \vec{m} \\
& =2 \sum_{p} \vec{b}(p) \times \delta \vec{g}_{q=0} .
\end{aligned}
$$

In the right-hand side, all terms that are coming from the explicit knowledge of the solution $\delta \vec{g}$ needed to evaluate this sum over the momentum-dependent spin-orbit term $\vec{b}$ are collected. The separation of the balance equation in this form has the merit to see already the collective spin mode structure. The fine details are then worked out when we know the explicit solution in Sec. III.

Since we have $\vec{m}=m \vec{e}_{Z}$ and $\vec{V}=V \vec{e}_{Z}$ the equation for the magnetization becomes

$$
\left(\begin{array}{ccc}
-i \omega & 2\left(N V+\mu_{B} B\right) & 0 \\
-2\left(N V+\mu_{B} B\right) & -i \omega & 0 \\
0 & 0 & -i \omega
\end{array}\right) \delta \vec{m}=2 \sum_{p} \vec{b} \times \delta \vec{g}_{q=0}
$$

neglecting the quadratic terms of the vector relaxation times $\vec{\tau}^{-1}$. The latter would add a term $-i\left(\vec{\tau}^{-1}+2 \vec{m} \times\right.$ $V) \vec{\tau}^{-1} \cdot \delta \vec{m} / \omega$ to the left-hand side.

Inverting 26 provides the solution of the magnetization change provided we know the solution of $\delta \vec{g}$ on the right hand side. Interestingly, this inversion is only possible for a nonzero determinant. The vanishing determinant provides therefore the eigenmodes of spin waves with some possible modifications due to the spin-orbit coupling term.

The dispersion relation from the condition of vanishing determinant in 26 yields the two spin waves

$$
\omega_{\text {spin }}= \pm 2\left|N V+\mu_{B} B\right|
$$

which shows the linear splitting due to the driving external magnetic field and the permanent magnetization $\vec{V}=V \vec{e}_{z}$.

\section{Solution of linearized coupled kinetic equations}

As we have seen, even the balance equations for the linearized kinetic equations 19 are not closed if we do not know the solution for $\delta \vec{g}$, which comes from the momentum dependence of $\vec{\Sigma}$. In the following we will present two ways to solve 19 . First the elementary direct way and secondly with the help of operator algebra. The latter is then applicable directly to solve the kinetic equation with magnetic fields in the next section.

\section{Solution with the help of vector equation}

Solving the first equation of $(19$ for

$$
\delta f=-\frac{1}{a}\left(\overrightarrow{\mathcal{B}} \cdot \delta \vec{g}-S_{0}\right)
$$

and introducing the result into the second equation leads to a vector equation of type $\mathrm{A} 6$ and with the help of the abbreviations

$$
\vec{c}=-\frac{\overrightarrow{\mathcal{B}}}{a}, \quad \vec{z}=\frac{\vec{\Sigma}}{a}, \quad \vec{o}=\frac{1}{a}\left(\vec{c} S_{0}+\vec{S}\right)
$$

and setting $B=\vec{o}, A=2 \vec{z}$, and $Q=-V=\vec{c}$ it can be readily solved [ see Eq. A8], which becomes

$$
\begin{aligned}
& \delta \vec{g}=\frac{\vec{o}+\vec{c} \times(\vec{c} \times \vec{o})+2(\vec{z} \times \vec{o})+4 \vec{z}(\vec{z} \cdot \vec{o})-2(\vec{z} \cdot \vec{c})(\vec{c} \times \vec{o})}{1-c^{2}+4 z^{2}-4(\vec{z} \cdot \vec{c})^{2}} \\
& \delta f=\vec{c} \cdot \vec{\delta} g+\frac{S_{0}}{a}
\end{aligned}
$$




\section{Solution with the help of operator algebra}

As a second possibility, we rewrite equations 19 with the help of the identity

$$
\begin{aligned}
& \vec{c} \cdot \vec{g}+(\vec{\sigma} \cdot \vec{c}) f-2 \vec{\sigma} \cdot(\vec{\sigma} \times \vec{g})= \\
& \vec{\sigma} \cdot \frac{\vec{c}+2 i \vec{\sigma}}{2} \hat{\rho}+\hat{\rho} \vec{\sigma} \cdot \frac{\vec{c}-2 i \vec{\sigma}}{2}
\end{aligned}
$$

into one operator equation for $\delta \hat{F}=\delta f+\vec{\sigma} \cdot \vec{\delta} g$ and transform the frequency back in time $-i \omega \rightarrow \partial_{t}$ :

$$
\begin{aligned}
& \hat{S}_{p}(t)=S_{0}(t)+\vec{\tau} \cdot \vec{S}(t)=\left(\partial_{t}+i \frac{p q}{m}+\tau^{-1}\right) \delta \hat{F}(t) \\
& +\left(\frac{\vec{b}}{2}+i \vec{\Sigma}\right) \cdot \vec{\sigma} \delta \hat{F}+\delta \hat{F}\left(\frac{\vec{b}}{2}-i \vec{\Sigma}\right) \cdot \vec{\sigma}
\end{aligned}
$$

This equation is easily solved

$$
\delta \hat{F}(t)=\int_{-\infty}^{t} d \bar{t} \mathrm{e}^{i\left(\frac{p q}{m}-\frac{i}{\tau}\right)(\bar{t}-t)} \mathrm{e}^{\left(\frac{\vec{b}}{2}+i \vec{\Sigma}\right) \cdot \vec{\sigma}(\bar{t}-t)} \hat{S}_{p}(\bar{t}) \mathrm{e}^{\left(\frac{\vec{b}}{2}-i \vec{\Sigma}\right) \cdot \vec{\sigma}(\bar{t}-t)}
$$

and transformed back in frequency space to obtain

$$
\delta \hat{F}(\omega)=\int_{-\infty}^{0} d x \mathrm{e}^{i\left(\frac{p q}{m}-\omega-\frac{i}{\tau}\right) x} \mathrm{e}^{\left(\frac{\vec{b}}{2}+i \vec{\Sigma}\right) \cdot \vec{\sigma} x} \hat{S}_{p}(\omega) \mathrm{e}^{(\vec{b}-i \vec{\Sigma}) \cdot \vec{\sigma} x}
$$

Further evaluation is presented in Appendix B. With the help of (B11) and (B12) we evaluate the corresponding integrals and all scalar terms determine $\delta f$, and all terms proportional to $\sigma$ determine $\vec{\delta} g$. We obtain again the result 3098

$$
\delta \vec{g}=\frac{\vec{o}+\vec{c} \times(\vec{c} \times \vec{o})+2(\vec{z} \times \vec{o})+4 \vec{z} \cdot(\vec{z} \cdot \vec{o})-2(\vec{z} \cdot \vec{c})(\vec{c} \times \vec{o})}{\left(1-c^{2}\right)\left(1+4 z^{2}\right)} .
$$

To see the known limits, we inspect some further approximations.

\section{Long-wavelength limit}

We assume only a scalar relaxation time and neglect therefore the vector part describing skew scattering and side jump effects. Further we use the long wavelength limit and expand in first orders of $q \partial_{p} \vec{\Sigma}$ which translates into $\vec{c}^{2} \approx 0$ in 35 . Here, we have abbreviated the form of the mean-field self-energy

$$
\begin{aligned}
& U=\Phi+\delta \Sigma_{0} \\
& \vec{U}=\delta \vec{\Sigma}
\end{aligned}
$$

and we used $e \vec{E}=-i \vec{q} \Phi, \bar{\omega}=i a=\omega-\vec{p} \cdot \vec{q} / m+i \tau^{-1}$ and the meanfields

$$
\delta \Sigma_{0}=V_{0} \delta n+\vec{V} \cdot \delta \vec{s}, \quad \delta \vec{\Sigma}=\vec{V} \delta n+V_{0} \delta \vec{s} .
$$

Then the solution 28 and 35 reads $[\vec{g}=g \vec{e}]$

$$
\begin{aligned}
& \delta f=-\frac{1}{\bar{\omega}}\left\{U q \partial_{p} f+\vec{U} q \partial_{p} \vec{g}-i \frac{\delta n}{\tau \partial_{\mu} n} \partial_{\mu} f-q \partial_{p} \vec{\Sigma} \cdot \vec{\delta} g\right\} \\
& \delta \vec{g}=\delta \vec{g}^{\mathrm{asy}}+\delta \vec{g}_{\mathrm{Rabi}}^{\mathrm{sym}}+\delta \vec{g}_{\mathrm{n}}^{\mathrm{sym}}
\end{aligned}
$$

Each of the terms corresponds to one of the three possible precession motions:

$$
\frac{1}{4|\Sigma|^{2}-\omega^{2}}\left(\begin{array}{c}
-i \omega \\
\frac{4 i|\Sigma|^{2}}{\omega} \\
2|\Sigma|
\end{array}\right)=\int_{0}^{\infty} \mathrm{e}^{i \omega t}\left(\begin{array}{c}
\cos 2|\Sigma| t \\
1-\cos 2|\Sigma| t \\
\sin 2|\Sigma t|
\end{array}\right) .
$$

The $\sin 2|\Sigma| t$ motion is responsible for the anomalous Hall effect and their terms are collected in $\delta \vec{g}^{\text {asy }}$. Let us write out the explicit forms. The symmetric solution consists of an frequency denominator with Rabi-satellites

$$
\begin{aligned}
\delta \vec{g}_{\mathrm{Rabi}}^{\mathrm{sym}}= & \frac{1}{2}\left(\frac{1}{\bar{\omega}-2 \Sigma}+\frac{1}{\bar{\omega}-2 \Sigma}\right)\left[-U g q \partial_{p} \vec{e}-2 i \vec{g} \times \delta \vec{\Sigma}\right. \\
& \left.+q \partial_{p} f \vec{e} \times(\vec{e} \times \vec{U})+i \frac{\delta n}{\tau \partial_{\mu} n} g \partial_{\mu} \vec{e}\right]
\end{aligned}
$$

and a part with a normal denominator $\bar{\omega}=\omega+i / \tau-$ $\vec{p} \vec{q} / m$ :

$$
\delta \vec{g}_{\mathrm{n}}^{\mathrm{sym}}=\frac{1}{\bar{\omega}}\left[-U \vec{e} q \partial_{p} g-q \partial_{p} f \vec{e} \times(\vec{e} \cdot \vec{U})+i \frac{\delta n}{\tau \partial_{\mu} n} \partial_{\mu} g \vec{e}\right]
$$

which can be combined together

$$
\begin{aligned}
\delta \vec{g}^{\mathrm{sym}} & =\frac{1}{\bar{\omega}\left(4|\vec{\Sigma}|^{2}-\bar{\omega}^{2}\right)}\left\{\left[\bar{\omega}^{2} \vec{U}-4|\vec{\Sigma}|^{2} \vec{e}(\vec{e} \cdot \vec{U})\right] q \partial_{p} f\right. \\
& +\bar{\omega}^{2}\left(U q \partial_{p} \vec{g}-i \frac{\delta n}{\tau \partial_{\mu} n} \partial_{\mu} \vec{g}\right) \\
& \left.-4 \Sigma^{2}\left(U q \partial_{p} g-i \frac{\delta n}{\tau \partial_{\mu} n} \partial_{\mu} g\right) \vec{e}-2 i \bar{\omega}^{2} \vec{U} \times \vec{g}\right\} .
\end{aligned}
$$

The term responsible for the anomalous Hall effect reads

$$
\begin{aligned}
& \delta \vec{g}^{\text {asy }}=\frac{i}{2}\left(\frac{1}{\bar{\omega}+2 \Sigma}-\frac{1}{\bar{\omega}-2 \Sigma}\right)\left\{\vec{e} \times \vec{U} q \partial_{p} f\right. \\
& \left.+\left[U \vec{e} \times q \partial_{p} \vec{e}-2 i \vec{e} \times(\vec{U} \times \vec{e})-i \frac{\delta n}{\tau \partial_{\mu} n} \vec{e} \times \partial_{\mu} \vec{e}\right] g\right\}
\end{aligned}
$$

In order to compare with the homogeneous solution presented in Eq. (143) of part I

$$
\begin{aligned}
\delta \vec{\rho}(\omega, k)= & \frac{i \omega}{4|\Sigma|^{2}-\omega^{2}} e E \partial_{k} \vec{g} \\
& -4 i \frac{1}{\omega\left(4|\Sigma|^{2}-\omega^{2}\right)} \vec{\Sigma}\left(\vec{\Sigma} \cdot e E \partial_{k} \vec{g}\right) \\
& -2 \frac{1}{4|\Sigma|^{2}-\omega^{2}} \vec{\Sigma} \times e E \partial_{k} \vec{g}
\end{aligned}
$$


we take the $q \rightarrow 0$ limit of 38 with $\vec{q} U=i e \vec{E}+$ $o(q), q \vec{U}=o(q)$ and obtain

$$
\begin{aligned}
\delta f= & -\frac{i}{\bar{\omega}}\left(e E \partial_{p} f-\frac{\delta n}{\tau \partial_{\mu} n} \partial_{\mu} f\right) \\
\delta \vec{g}^{\text {sym }}= & -\frac{i \bar{\omega}}{\bar{\omega}^{2}-4|\Sigma|^{2}}\left(e E \partial_{p} \vec{g}-\frac{\delta n}{\tau \partial_{\mu} n} \partial_{\mu} \vec{g}-2 \vec{U} \times \vec{g}\right) \\
& +\frac{4 i \Sigma^{2}}{\bar{\omega}\left(\bar{\omega}^{2}-4|\Sigma|^{2}\right)}\left(e E \partial_{p} g-\frac{\delta n}{\tau \partial_{\mu} n} \partial_{\mu} g\right) \vec{e} \\
\delta \vec{g}^{\text {asy }}= & \frac{2|\Sigma| g}{\bar{\omega}^{2}-4|\Sigma|^{2}}\left[\vec{e} \times e E \partial_{p} \vec{e}-2 \vec{e} \times(\vec{U} \times \vec{e})\right. \\
& \left.-\frac{\delta n}{\tau \partial_{\mu} n} \vec{e} \times \partial_{\mu} \vec{e}\right] .
\end{aligned}
$$

Without vector meanfield variation $\vec{U} \approx 0$ and relaxation time, the last term responsible for the anomalous Hall effect corresponds directly to the third one in Eq. (44). The first two terms correspond to the first two ones in Eq. (44) as simple algebra shows observing that $\vec{\Sigma} \cdot \partial \vec{e}=0$ since $\vec{e}=\vec{\Sigma} /|\vec{\Sigma}|, \vec{e}(\vec{e} \cdot \partial \vec{g})=\vec{e} \partial g$ and $\vec{e} \times(\vec{e} \times \partial \vec{g})=$ $-g \partial \vec{e}$. The term $\vec{e} \times e E \partial_{p} \vec{e}$ of Eq. 45 corresponds to the precession term found in Ref. ${ }^{31}$ as an additional rotation of the magnetization.

\section{Response functions}

We want now to integrate the linearized solution 40, (41) and (43) over the momentum to obtain the density and spin response functions including the meanfield and spin-orbit coupling effects. This will lead to a selfconsistent equation. We can design the dimensionality of the considered problem as done above after Eq. (1).

The final result for the particle and spin density response using only intrinsic meanfields $\delta \Sigma_{0}=V_{0} \delta n+\vec{V} \cdot \delta \vec{s}$ and $\delta \vec{\Sigma}=\vec{V} \delta n+V_{0} \delta \vec{s}$ leads to the following linear system

$$
\begin{aligned}
& \left(1-\Pi_{0} V_{0}-\vec{\Pi} \cdot \vec{V}+\frac{i \Pi_{0 \mu}}{\tau \partial_{\mu} n}\right) \delta n=\Pi_{0} \Phi+\left(\Pi_{0} \vec{V}+\vec{\Pi} V_{0}\right) \cdot \delta \vec{s} \\
& \left(1-\Pi_{0} V_{0}-\overleftrightarrow{\Pi} V_{0}\right) \delta \vec{s}=\vec{\Pi}_{3} \Phi+\vec{\Pi}_{3}(\vec{V} \cdot \delta \vec{s})+V_{0} \vec{\Pi}_{2} \times \delta \vec{s} \\
& +\left(V_{0} \vec{\Pi}_{3}+\Pi_{0} \vec{V}+\vec{\Pi}_{2} \times \vec{V}+\overleftrightarrow{\Pi} \cdot \vec{V}-\frac{i}{\tau \partial_{\mu} n} \vec{\Pi}_{\partial}\right) \delta n
\end{aligned}
$$

with the abbreviations for the polarizations

$$
\begin{aligned}
\vec{\Pi}_{2} & =\vec{\Pi}_{g}+\vec{\Pi}_{x f} \\
\vec{\Pi}_{3} & =\vec{\Pi}+\vec{\Pi}_{x g}+\vec{\Pi}_{e} \\
\vec{\Pi}_{\partial} & =\vec{\Pi}_{x \mu}+\vec{\Pi}_{\mu}+\vec{\Pi}_{\mu e} \\
\overleftrightarrow{\Pi} & =\overleftrightarrow{\Pi}_{f e}+\overleftrightarrow{\Pi}_{x e} .
\end{aligned}
$$

The $1 / \tau$ terms come from the Mermin conserving relaxation-time approximation which means a relaxation towards a local equilibrium specified such that local conservation laws are obbeyed ${ }^{74 / 75 / 77}$.
It was helpful here to define the polarization functions according to the three precessions expressed by the parts (40), (41) and (43). The standard polarization functions for scalar and vector distribution coming from 40 read with $\bar{\omega}=\omega-\vec{p} \cdot \vec{q} / m+i / \tau$

$$
\begin{aligned}
\Pi_{0}(q \omega) & =-\sum_{p} q \partial_{p} f \frac{1}{\bar{\omega}} \\
\Pi_{0 \mu}(q \omega) & =-\sum_{p} \partial_{\mu} f \frac{1}{\bar{\omega}} \\
\vec{\Pi}(q \omega) & =-\sum_{p} q \partial_{p} \vec{g} \frac{1}{\bar{\omega}} \\
\vec{\Pi}_{\mu}(q \omega) & =-\sum_{p} \partial_{\mu} \vec{g} \frac{1}{\bar{\omega}} .
\end{aligned}
$$

The remaining parts of 40 and (41) combine into the forms of

$$
\frac{4 \Sigma^{2}}{\bar{\omega}\left(4 \Sigma^{2}-\bar{\omega}^{2}\right)}=\frac{1}{\bar{\omega}}-\frac{1}{2}\left(\frac{1}{\bar{\omega}+2 \Sigma}+\frac{1}{\bar{\omega}-2 \Sigma}\right)
$$

which vanish quadratically with the vector meanfield

$$
\begin{aligned}
\vec{\Pi}_{e}(q \omega) & =\sum_{p} g q \partial_{p} \vec{e} \frac{4 \Sigma^{2}}{\bar{\omega}\left(4 \Sigma^{2}-\bar{\omega}^{2}\right)} \\
\vec{\Pi}_{\mu e}(q \omega) & =\sum_{p} g \partial_{\mu} \vec{e} \frac{4 \Sigma^{2}}{\bar{\omega}\left(4 \Sigma^{2}-\bar{\omega}^{2}\right)} \\
\overleftrightarrow{\Pi}_{f e}(q \omega) & =\sum_{p} q \partial_{p} f(1-\vec{e} \circ \vec{e}) \frac{4 \Sigma^{2}}{\bar{\omega}\left(4 \Sigma^{2}-\bar{\omega}^{2}\right)}
\end{aligned}
$$

and a rotation part

$$
\vec{\Pi}_{g}(q \omega)=-i \sum_{p} \vec{g}\left(\frac{1}{\bar{\omega}+2 \Sigma}+\frac{1}{\bar{\omega}-2 \Sigma}\right) .
$$

The responses from the asymmetric part (43) lead to

$$
\begin{aligned}
\vec{\Pi}_{x f}(q \omega) & =i \sum_{p} \vec{e} q \partial_{p} f \frac{1}{2}\left(\frac{1}{\bar{\omega}+2 \Sigma}-\frac{1}{\bar{\omega}-2 \Sigma}\right) \\
\vec{\Pi}_{x g}(q \omega) & =i \sum_{p} \vec{e} \times q \partial_{p} \vec{g} \frac{1}{2}\left(\frac{1}{\bar{\omega}+2 \Sigma}-\frac{1}{\bar{\omega}-2 \Sigma}\right) \\
\vec{\Pi}_{x \mu}(q \omega) & =i \sum_{p} \vec{e} \times \partial_{\mu} \vec{g} \frac{1}{2}\left(\frac{1}{\bar{\omega}+2 \Sigma}-\frac{1}{\bar{\omega}-2 \Sigma}\right) \\
\overleftrightarrow{\Pi}_{x e}(q \omega) & =2 \sum_{p} g(1-\vec{e} \circ \vec{e}) \frac{1}{2}\left(\frac{1}{\bar{\omega}+2 \Sigma}-\frac{1}{\bar{\omega}-2 \Sigma}\right)
\end{aligned}
$$

which vanish linearly in orders of the self-energy. In the case of vanishing spin-orbit coupling, i.e. no momentum dependence of $\vec{e}$, we have $\vec{\Pi}_{x g}=\vec{\Pi}_{x \mu}=\vec{\Pi}_{e}=\vec{\Pi}_{\mu e}=0$.

It is known that vertex corrections lead to additional structure factors in the RPA polarization functions, which would extend the expressions here. Here, it 
is shown that the spin-orbit coupling causes a zoo of additional RPA polarization forms even on the level of the single-loop approximation, which is the highest level to be obtained by the linearization of the mean-field equations.

\section{SPIN AND DENSITY WAVES}

The system of density and spin responses (46) allows us to determine the spin and density waves that might be excited in the system. It is convenient to continue to work in the wave number space $r \leftrightarrow q$. For the magnetization and total particle number (23), we consider only dipole modes that are characterized by the first-order moments

$$
\begin{aligned}
& \Psi_{j}=\int d^{3} r x_{j} \delta n(r)=-\left.i \partial_{q_{j}} \delta n_{q}\right|_{q=0} \\
& \vec{\Psi}_{j}=\int d^{3} r x_{j} \delta \vec{s}(r)=-\left.i \partial_{q_{j}} \delta \vec{s}_{q}\right|_{q=0}
\end{aligned}
$$

The linear response for dipole modes is assumed to be characterized by linear deviations $\delta f=\overrightarrow{\beta_{1}} \cdot \overrightarrow{\partial_{r}} f_{0}+\overrightarrow{\beta_{2}} \cdot \overrightarrow{\partial_{p}} f_{0}$ and $\delta g=\vec{\alpha}_{1} \cdot \overrightarrow{\partial_{r}} g_{0}+\vec{\alpha}_{2} \cdot \overrightarrow{\partial_{p}} g_{0}$ such that we have

$$
\begin{aligned}
\left.\delta n\right|_{q=0} & =\left.i \vec{\beta}_{1} \cdot \vec{q} n\right|_{q=0}=0 \\
\left.\delta \vec{s}\right|_{q=0} & =\left.i \vec{\alpha}_{1} \cdot \vec{q} \vec{s}\right|_{q=0}=0
\end{aligned}
$$

for the long-wavelength limit of the deviations themselves. In order to determine the eigenmodes of (46), we do not need the actual values of $\alpha$ and $\beta$. Due to the properties (53) and (54), we can apply the $q$-derivative directly to (46) and obtain an equation system where $\delta n$ and $\delta \vec{s}$ are replaced by $\Psi_{j}$ and $\vec{\Psi}_{j}$ in 46 and all response functions have to be taken in the $q \rightarrow 0$ limit. Any derivative of the latter vanishes since they are connected with terms (54). This simplifies the analysis appreciably and shows the strength of the response system (46). For quadrupole modes where the second derivative is needed, one has to calculate also the $q \rightarrow 0$ limit of the derivatives of the polarization functions.

Further analysis relays on the expansion of different polarization functions as presented in Appendix C. Then, the momentum integration still cannot be performed analytically if the momentum-dependent spin-orbit term $b(p)$ in $\vec{\Sigma}$ is present. Therefore we treat them linearly in $\vec{b}(p)$, which allows to give an explicit form. In fact, these terms are only present if the spin-orbit coupling creates an explicit $p$-dependence in $\vec{\Sigma}=\vec{\Sigma}_{n}+\vec{b}(p)$, where we denote the momentum-independent selfenergy with $\vec{\Sigma}_{n}=n \vec{V}+V_{0} \vec{s}+\mu_{B} \vec{B}$. Let us define the $z$-direction by this last term $\vec{e}_{z}=\vec{\Sigma}_{n} /\left|\vec{\Sigma}_{n}\right|$ and expand all directions in first order of $\vec{b}(p)$.

The direction of effective polarization becomes

$$
\vec{e}=\frac{\vec{\Sigma}}{|\Sigma|}=\vec{e}_{z}\left(1-\frac{b_{\perp}^{2}}{2}\right)+\vec{b}_{\perp}\left(1-b_{3}\right)
$$

where we use the short-hand notation

$$
\frac{\vec{b}_{p}}{\Sigma_{n}}=\vec{b}_{\perp}+\vec{e}_{z} b_{3}
$$

Since the distribution functions in equilibrium are functions of $|\vec{\Sigma}|$ according to 16 , i.e. functions of $b_{\perp}^{2}$ and $b_{3}$, and since the latter ones are even in momentum direction, the distributions are even in momentum direction as well. Therefore the polarization becomes

$$
\vec{s}=\sum_{p} g \vec{e}=\vec{e}_{z} \sum_{p} g\left(1-\frac{b_{\perp}^{2}}{2}\right)=\vec{e}_{z}\left(s_{0}-\frac{B_{g}^{2}}{2}\right)
$$

with

$$
s_{0}=\sum_{p} g ; \quad B_{g}^{2}=\sum_{p} b_{\perp}^{2} g ; \quad m=s_{q=0} .
$$

The $q \rightarrow 0$ limit is of course dependent on the $q$ dependence of the potential. Therefore let us analyze the neutral scattering $V_{0}$ and charged scattering $V_{0}=e^{2} / \epsilon q^{2}$ separately. As we will see, only the latter provides density waves as a collective plasma oscillation.

\section{A. Excitation with neutral scattering}

To study the excitation modes for scattering with neutral impurities, we can restrict ourselves to the lowestorder expansion in $q$, which simplifies the results of the last section again. Especially $\Pi_{0}=\vec{\Pi}=\vec{\Pi}_{x f}=\vec{\Pi}_{x g}=$ $\vec{\Pi}_{e}=\overleftrightarrow{\Pi}_{f e}=0$. According to $\sqrt{53}$ and 54 we obtain for the density excitation from 46

$$
\left(1-\frac{i}{\omega_{+} \tau}+o\left(q^{2}\right)\right) \Psi_{j}=0
$$

with $\omega^{+}=\omega+i / \tau$ which means that we have either the zero mode $\omega=0$ or $\Psi_{j}=0$, i.e., no density dipole wave excitations. This will be different when we consider charged scattering in the next section.

With the help of this result the equation for the spin excitation becomes from 46 in long-wavelength expansion simply

$$
\mathcal{D} \vec{\Psi}_{j} \equiv\left(1-V_{0} \overleftrightarrow{\Pi}\right) \vec{\Psi}_{j}-V_{0} \vec{\Pi}_{2} \times \vec{\Psi}_{j}=0
$$

with

$$
\begin{aligned}
\vec{\Pi}_{2} & =i \vec{e}_{z}\left[-s_{0}+\frac{B_{g}^{2}}{2}\left(1-\Sigma_{n} \partial_{\Sigma_{n}}\right)-\frac{q^{2} E_{g}}{D m_{e}} \partial_{\omega}^{2}\right] \frac{2 \omega}{\omega^{2}-4 \Sigma_{n}^{2}} \\
\overleftrightarrow{\Pi} & =\left(\begin{array}{ccc}
s_{0}+B_{g 11}+\frac{B_{g}^{2} \Sigma_{n}}{2} \partial_{\Sigma_{n}} & 0 & 0 \\
0 & s_{0}+B_{g 22}+\frac{B_{g}^{2} \Sigma_{n}}{2} \partial_{\Sigma_{n}} & 0 \\
0 & 0 & -B_{g}^{2}
\end{array}\right) \\
& \times \frac{4 \Sigma_{n}}{4 \Sigma_{n}^{2}-\omega_{+}^{2}} .
\end{aligned}
$$




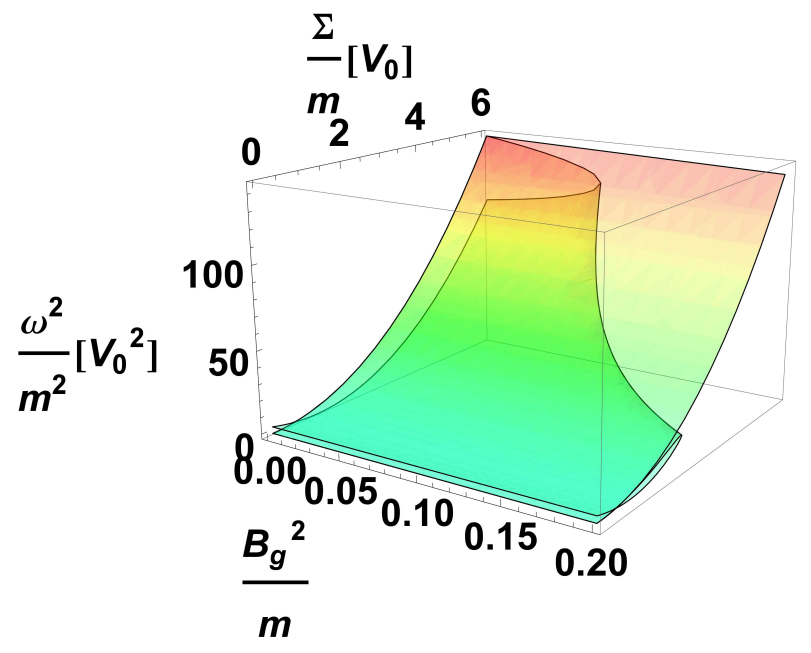

FIG. 1: The three frequencies of spin excitation mode 63 as a function of selfenergy $\Sigma$ and thermally averaged spin-orbit coupling 58 and magnetization $m=s_{q=0}$.

The vanishing determinant of $\mathcal{D}$ in $(60)$ yields the collective spin excitation wave.

Neglecting first the thermally averaged spin-orbit terms completely we obtain the modes

$$
\omega_{\text {spin }}=-\frac{i}{\tau} \pm 2\left|s V_{0}-\Sigma_{n}\right|
$$

which shows that two spin modes are excited. Provided we have an interaction $V_{0}$ the mode is shifted simply by the meanfield selfenergy $\Sigma_{n}=n V+V_{0} s+\mu_{B} B$ and this mode $\omega \sim 2 \mu_{B} B^{\text {eff }}$ is exclusively dependent on the effective Zeeman shift $\mu_{B} B^{\text {eff }}=n V+\mu_{B} B$. This result we had already obtained as zero order in spin-orbit coupling (27) in agreement with the recent report of a transition from charge to spin density waves only appearing at a finite Zeeman field 34 .

As discussed in Sec. III.H of part I of this paper [], the selfconsistency would result into the replacement $\Sigma_{n}=$ $n V+V_{0} s+\mu_{B} B \rightarrow\left(n V+\mu_{B} B\right) /\left(1+\frac{m_{e}}{2 \pi \hbar^{2}} V_{0}\right)$. In order to facilitate the following notation we write shortly $\Sigma$ for this selfconsistent $\Sigma_{n}$.

The dispersion including the spin-orbit coupling is dependent on the parameter $B_{g}^{2}=B_{g 11}^{2}+B_{g 22}^{2}$ given in $(58)$. The spin modes as zeros of $|\mathcal{D}|$ of $(60)$ appear to be

$$
\begin{aligned}
& \left(\omega+\frac{i}{\tau}\right)^{2}-4\left(\Sigma-s V_{0}\right)^{2}=2 V_{0}\left[B_{g}^{2} \Sigma\right. \\
& \left.+\left(s V_{0}-2 \Sigma\right)\left(s \pm \sqrt{s^{2}-\frac{2}{V_{0}} \Sigma B_{g}^{2}}\right)\right]
\end{aligned}
$$

together with a third mode as the sum of the right side. The result is plotted in Fig. 1. One sees that the two modes 62 appear, which differ with increasing $\Sigma$. The threefold splitting of spin modes was reported in Ref. ${ }^{71}$.

A closer inspection shows that one mode can become imaginary for small selfenergies $\Sigma$. In fact, expanding up

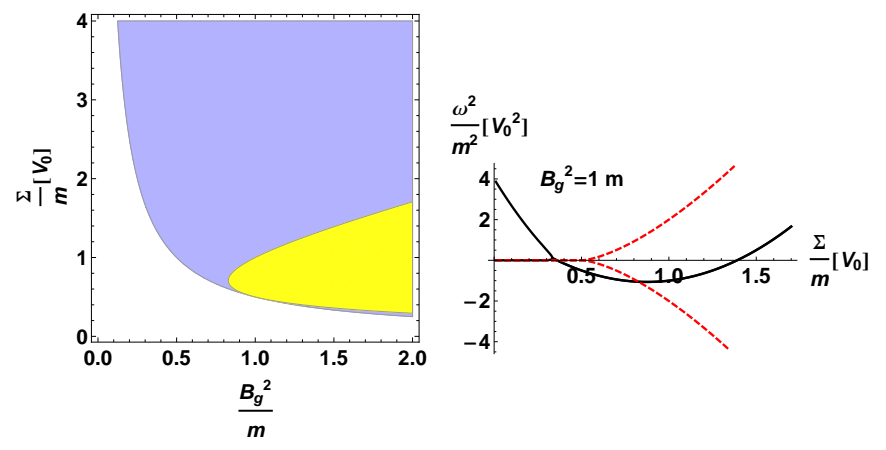

FIG. 2: Left: The maximal range of spin-wave instability for vanishing collisional damping. The outer area (blue) designs the $\operatorname{Im} \omega^{2} \neq 0$ modes and the inner (yellow) area the $\omega^{2}<0$ modes. Right: The real (black solid) and imaginary (dashed red) part of $\omega^{2}$ versus $\Sigma$

to $+o\left(B_{\perp}^{2},\right)$ one gets

$$
\left(\omega_{\text {spin }}+\frac{i}{\tau}\right)^{2}=4\left\{\begin{array}{c}
\Sigma^{2}\left(1-\frac{B_{g}^{2}}{s}\right) \\
\left(\Sigma-s V_{0}\right)^{2}+\frac{B_{g}^{2} \Sigma\left(\Sigma-s V_{0}\right)}{s}
\end{array}\right.
$$

where the square of the first mode can become negative which means an imaginary mode. Due to the six-order polynomial in $\omega$, for each dispersion, also the complex conjugated one is a solution. A finite imaginary part means instability when it overcomes the damping by collisions $1 / \tau$.

The maximal range of such possible spin-wave instability (without collisional damping $1 / \tau \rightarrow 0$ ) is shown in Fig. 2. Here, we distinguish the region of $\omega^{2}<0$ appearing as the inner region (yellow) and the region where $\operatorname{Im} \omega^{2} \neq 0$ as the outer (blue) one. The twofold regions of complex frequencies are seen in the cut in Fig. 2.

The physics of these instabilities can be seen more explicitly in the zero temperature limit where in quasitwo-dimensions and in the presence of linear Dresselhaus $\beta=\beta_{D}$ or Rashba $\beta=\beta_{R}$ spin-orbit coupling the density and the polarization become

$$
\begin{aligned}
n & =\sum_{p} f=\frac{m_{e}}{2 \pi \hbar^{2}}\left(\epsilon_{f}+\epsilon_{\beta}\right) \\
s & =\sum_{p} g=-\frac{m_{e}}{2 \pi \hbar^{2}} \sqrt{\epsilon_{\beta}\left(\epsilon_{\beta}+2 \epsilon_{f}\right)+\Sigma_{n}^{2}}
\end{aligned}
$$

with the spin-orbit energy $\epsilon_{\beta}=m_{e} \beta^{2}$. Further we have

$$
B_{g}^{2}=\frac{4 \pi \hbar^{2}}{m_{e}} p n^{2} \frac{\epsilon_{\beta}}{\Sigma_{n}^{2}}
$$

with the polarization $p=s / n$.

In Fig. 3 we plot the non-self-consistent and selfconsistent modes in dependence on the density. Using the polarization and the scaling with the Fermi energy $\epsilon_{f}$ allows us to get rid of the spin-orbit energy. One sees that the selfconsistency leads to smaller modes at smaller 

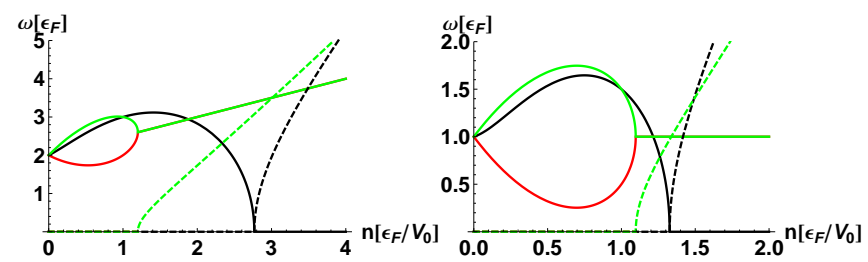

FIG. 3: The real (solid) and imaginary (dashed) part of $\omega$ as a function of the density for the effective magnetic field $\mu_{B} B^{\text {eff }}=n V+\mu_{B} B=1 \epsilon_{f}$, the polarization $p=0.5$ and the dimensionless potential $v_{0}=m_{e} V_{0} / 2 \pi \hbar^{2}=1$ at zero temperature. Left figure shows the selfconsistent result and right figure the non-selfconsistent one. Different branches of the single mode are distinguished additionally by different colors.

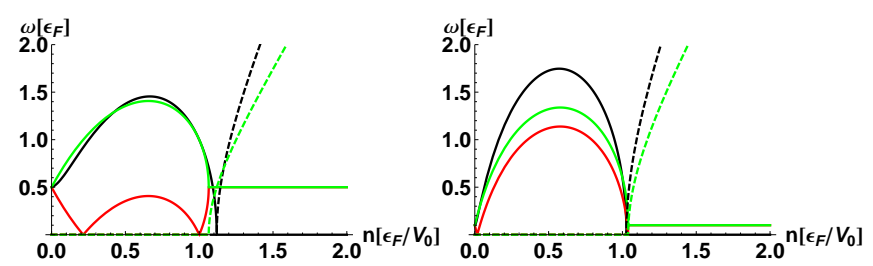

FIG. 4: The selfconsistent modes of figure 3 for effective magnetic fields $\mu_{B} B^{\text {eff }}=n V+\mu_{B} B=0.5 \epsilon_{f}$ (left) and $\mu_{B} B^{\text {eff }}=n V+\mu_{B} B=0.1 \epsilon_{f}$ (right).

densities. Up to a critical density, we do not have any imaginary part. As soon as the two spin modes vanish, a damping occurs that is symmetric in sign such that it denotes an instability. The third mode of 63 is vanishing at higher densities.

The dependence on the effective magnetic field is seen in Fig. 4, which shows that the two spin modes become nontrivial and vanish at the same density as the third mode for vanishing magnetic field.

The expansion of 63 in small spin-orbit coupling reads

$$
\left(\omega_{\text {spin }}+\frac{i}{\tau}\right)^{2}=\left\{\begin{array}{c}
4 \Sigma^{2}-8 \epsilon_{f} \epsilon_{\beta} \\
\left(4 \Sigma^{2}+8 \epsilon_{f} \epsilon_{\beta}\right)\left(1+\frac{m_{e}}{2 \pi \hbar^{2}} V_{0}\right)^{2}+o\left(\epsilon_{\beta}^{2}\right)
\end{array}\right.
$$

and the third mode $4 \Sigma^{2}-\frac{8 m_{e} V_{0}}{2 \pi \hbar^{2}} \epsilon_{f} \epsilon_{\beta}$. This shows that the spin modes becomes

$$
\omega+i / \tau=\left\{\begin{array}{c}
i\left(2 \beta p_{f}-\frac{\Sigma^{2}}{\beta p_{f}}\right) \\
\left(2 \beta p_{f}+\frac{\Sigma^{2}}{\beta p_{f}}\right)\left(1+\frac{m_{e} V_{0}}{2 \pi \hbar^{2}}\right)
\end{array}+o\left(\epsilon_{\beta}^{2}, \Sigma^{4}\right)\right.
$$

for small effective Zeeman fields providing a linear dependence of the energy and damping on $\beta$. This is in contrast to the influence of the Landau levels which provides quadratic dependencies $25 \mid 26$.

\section{B. Excitation with charged scattering}

Now we consider the charged scattering with an impurity Coulomb potential $V_{0}=e^{2} / \epsilon_{o} q^{2}$ or the scattering between charged particles as meanfields and in the relaxation time approximation. Using the long-wavelength expansions of Appendix C, we obtain the following equation system from 46

$$
\begin{aligned}
& \left(\frac{\omega_{+}^{2}}{\omega_{p}^{2}}+1-\frac{i \omega_{+}}{\omega_{p}^{2} \tau}\right) \Psi_{j}=\vec{m} \cdot \vec{\Psi}_{j} \\
& 2 \sum_{p} g(1-\vec{e} \circ \vec{e}) \vec{\Psi}_{j}+i \omega \vec{m} \times \vec{\Psi}_{j}=0
\end{aligned}
$$

with $\vec{m}=\vec{s}_{q=0}=\vec{e}_{z} s$. We used only the most divergent terms $\sim 1 / q^{2}$ in the equation for $\delta \vec{s}$ and have introduced the plasma frequency $\omega_{p}^{2}=e^{2} n / \epsilon_{0} m_{e}$. The eigenmodes of 69 can be seen to decouple for density and spin modes since the equation provides the eigenmode as a vanishing determinant of the matrix in front of $\vec{\Psi}_{j}$. The density eigenmodes are given by the vanishing left side of the first equation which implies $\vec{m} \perp \vec{\Psi}_{j}$ which means that we have only transverse spin modes with respect to the effective magnetization axes $\vec{m}$ of (57). The frequency of the density modes is just the damped plasma oscillation,

$$
\omega_{n}=-\frac{i}{2 \tau} \pm \sqrt{\omega_{p}^{2}-\frac{1}{4 \tau^{2}}}
$$

and the spin oscillation becomes

$$
\omega_{\text {spin }}=-\frac{i}{\tau} \pm 2\left[\Sigma+o\left(b^{2}\right)\right]
$$

where we have used the linear expansion in $b$ of the last paragraph. Compared with the spin mode of the neutral excitation 62 , we see that the term $s V_{0}$ is absent.

\section{Dielectric function}

The response function 13 describes the density change with respect to the external potential $\delta n=$ $\chi \Phi$ while the polarization function $\delta n=\Pi \Phi^{\text {ind }}$ is the density variation with respect to the induced potential $\Phi^{\text {ind }} V_{q} \delta n+\Phi$. Therefore one has $\chi=\Pi /\left(1-V_{q} \Pi\right)$, and the dielectric function as a ratio of the induced to the external potential is

$$
\frac{1}{\epsilon}=1+V_{q} \chi
$$

If we expand the response function up to quadratic orders of the wave vector, as performed in appendix C, the result for the dielectric function can be compactly written as

$$
\epsilon(\omega, q)=1-\frac{1}{\frac{1}{1-\epsilon(\omega, 0)}-\frac{q^{2}}{\kappa^{\mathrm{eff}}(\omega)}}
$$

where the long-wavelength dielectric function can be expressed as

$$
\begin{aligned}
& \epsilon(\omega, 0)=\epsilon_{\omega} \\
& +p^{2}\left(1-\frac{1}{\epsilon_{\omega}}\right)\left[1-\epsilon_{\omega}-\frac{B_{f}^{2}}{\epsilon_{\omega}}\left(\left(1-\epsilon_{\omega}\right)^{2}-\frac{\omega_{p}^{2}}{\omega^{2}\left(1+p^{2}\right)}\right)\right]
\end{aligned}
$$




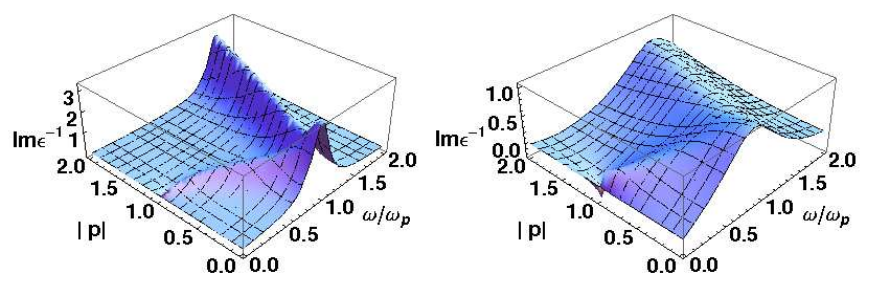

FIG. 5: The long-wave excitation function (74) for a collision frequency $\tau^{-1}=0.3 \omega_{p}$ (left) and $\tau^{-1}=1 \omega_{p}$ (right).

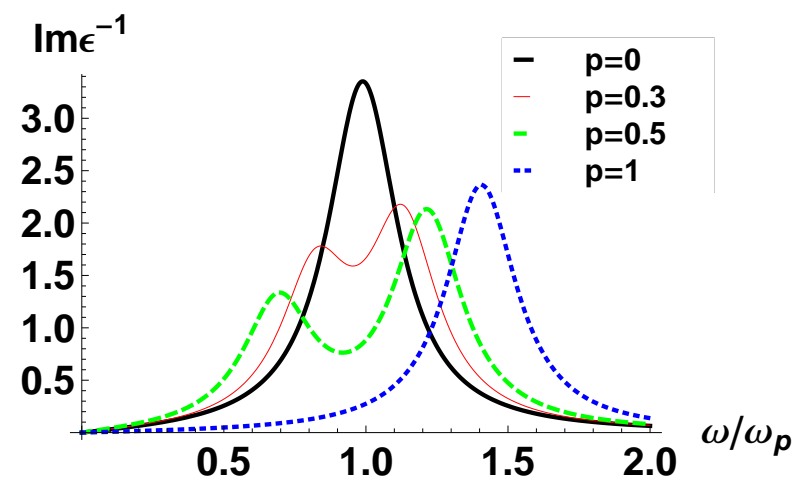

FIG. 6: The long-wave excitation function 74 for different polarizations versus frequency as cuts of figure 5 for $\tau^{-1}=$ $0.3 \omega_{p}$.

in terms of the Drude's expression

$$
\epsilon_{\omega}=1-\frac{\omega_{p}^{2}}{\omega\left(\omega+\frac{i}{\tau}\right)}
$$

and the effective polarization

$$
p=\frac{s}{n}=\frac{n_{\uparrow}-n_{\downarrow}}{n}-\frac{B_{g}^{2}}{2 n} .
$$

Here we use the zero temperature result for linear spinorbit coupling

$$
B_{g}^{2}=\frac{2 p}{1+p^{2}} B_{f}^{2}
$$

with (58). Let us discuss the long-wavelength limit of the dielectric function first without spin-orbit coupling $B_{f}=0$ but finite polarization 76 . This corresponds to the treatments of two-fluid models, e.g. one-dimensional quantum wires $\sqrt{82}$, with finite polarization. In figure 5 we plot the excitation function which yields the weight of the collective modes as a function of frequency and polarization. There we plotted a larger range of polarizations. Since the latter one is an effective one according to 76 we can have values smaller than -1 . A smaller relaxation time leads to a higher damping of modes, of course.

We see the appearance of two collective modes with increasing polarization. The plasma mode becomes split in a fast decaying mode with increasing polarization and

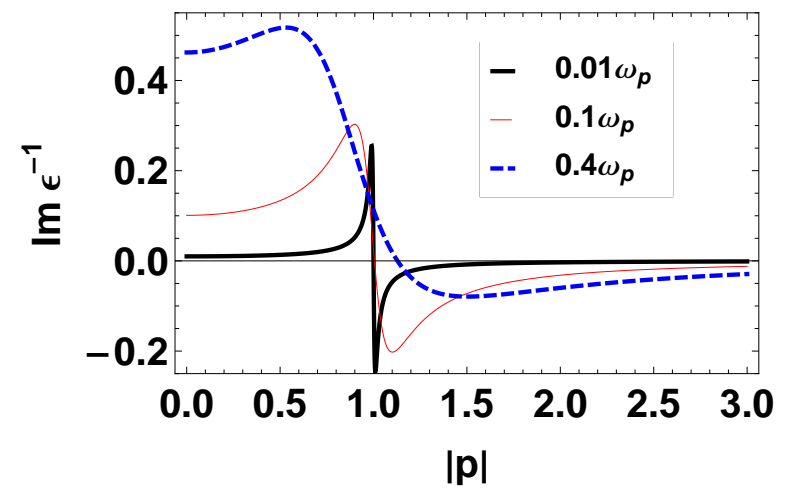

FIG. 7: The long-wave excitation function (74) for three small frequencies versus effective polarization 76 .

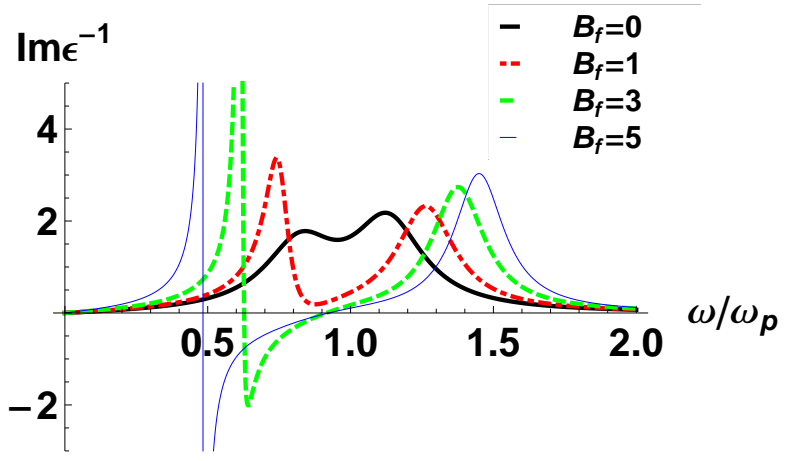

FIG. 8: The long-wave excitation function (74) for different spin-orbit couplings $B_{f}$ versus frequency with a fixed polarization $p=0.3$ of figure 6

a mode which becomes sharper again with increasing polarization. This is illustrated in figure 6 as cuts for special polarizations.

Near the point of vanishing first mode around $p=1$ the excitation function becomes negative indicating an instability. This is illustrated in the next figure 7. The effective polarization as the sum of polarization and spinorbit coupling term $B_{g}$ can lead to negative excitation functions indicating an instability. We will interpreted this instability as a de-mixing of spin states later when discussing the screening length.

Next we consider the influence of the spin-orbit coupling $B_{f}$ which is given in figure 8 for different values and a fixed polarization. We see that the spin-orbit coupling has basically the same effect as an additional polarization. Above a certain spin-orbit coupling the excitation function becomes negative indicating an instability. This is also visible in the figure 9 .

The range where the excitation function becomes negative indicating an instability is plotted in the next figure 10. This range indicates a spin domain separation and becomes large for increasing spin-orbit coupling parameter $B_{f}^{2}$ of C6. We interpret this as spin segregation as 


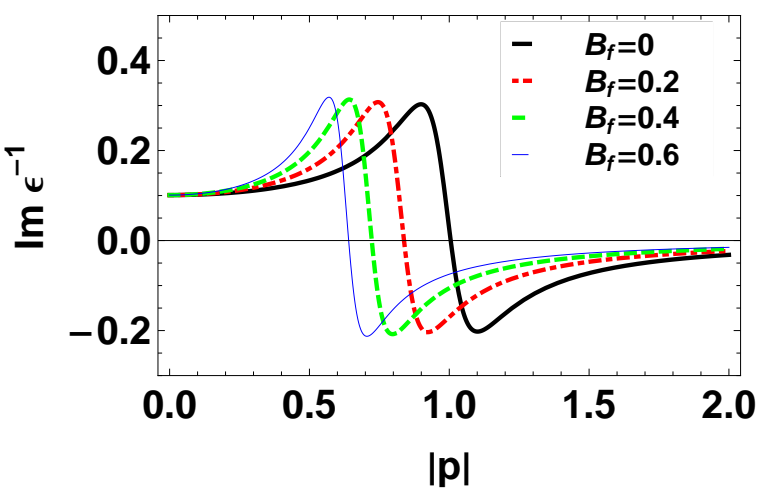

FIG. 9: The long-wave excitation function 74 for different spin-orbit couplings $B_{f}$ versus effective polarization $(76)$ with a fixed polarization $|p|=1$ and frequency $\omega=0.1 \omega_{p}$ of figure 7
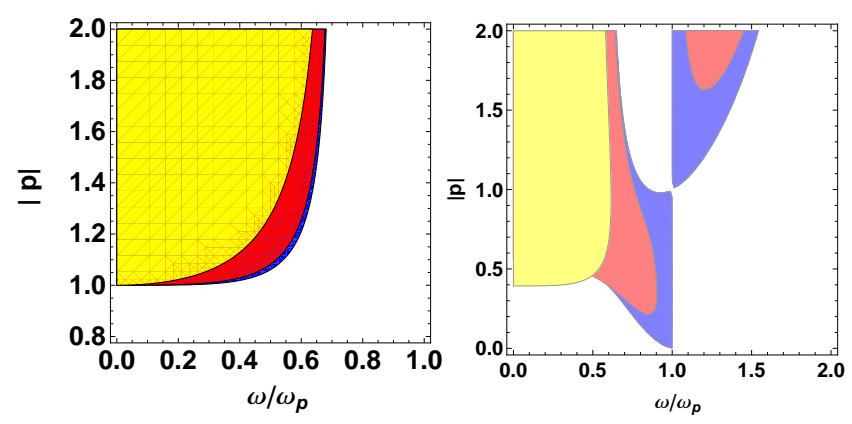

FIG. 10: The region where the excitation function becomes negative versus frequency and polarization for $B_{f}=0$ (left) and $B_{f}=2$ (right). The upper range (yellow) is for $1 / \tau=$ $1 \omega_{p}$, the middle (red) for $1 / \tau=0.3 \omega_{p}$ and the bottom (blue) one for $1 / \tau=0.001 \omega_{p}$.

observed in 17 and described in 19 .

a. Screening length Next we discuss the effective dynamical screening length $\chi^{\text {eff }}(\omega)$ of 73 which can be expressed shortly in terms of 75 as

$$
\begin{aligned}
& \left(\frac{\kappa}{\kappa^{\mathrm{eff}}(\omega)}\right)^{2}=\frac{1}{1-i \omega \tau}-p \kappa_{V}^{2} \frac{\epsilon_{\omega}-1}{\epsilon_{\omega}}\left\{1+\frac{1}{\left(1-\epsilon_{\omega}\right)\left(1-q_{\omega}\right)}\right. \\
& +B_{f} \frac{\epsilon_{\omega}-1}{\epsilon_{\omega}\left(1-q_{\omega}\right)}\left[1+\left(1+\frac{2(1-i \omega \tau)}{\omega_{p}^{2} \tau^{2}\left(\epsilon_{\omega}\left(1+q_{\omega}\right)-1\right)}\right)\right. \\
& \left.\left.\times\left(\frac{1}{\left(1-\epsilon_{\omega}\right)\left(1-q_{\omega}\right)}-q_{\omega}\right)\right]\right\}
\end{aligned}
$$

and the short-hand notation $q_{\omega}=p^{2}\left(\epsilon_{\omega}-1\right) / \epsilon_{\omega}$. The static limit where $\left(\epsilon_{\omega}-1\right) / \epsilon_{\omega} \rightarrow 1$ and $\epsilon_{\omega} \rightarrow \infty$ reads therefore

$$
\left(\frac{\kappa}{\kappa^{\mathrm{eff}}(0)}\right)^{2}=1-p \kappa_{V}^{2}\left[1+B_{f}\left(1-\frac{2 p^{2}}{\omega_{p}^{2} \tau^{2}\left(1-p^{4}\right)}\right)\right] .
$$

We have abbreviated $\kappa_{V}^{2}=\left(V+\mu_{B} B / n\right) \partial_{\mu} n=$ $\mu_{B} B^{\text {eff }} \partial_{n} n / n$. This result is explicitly an analytic longwavelength expression of the influence of polarization on

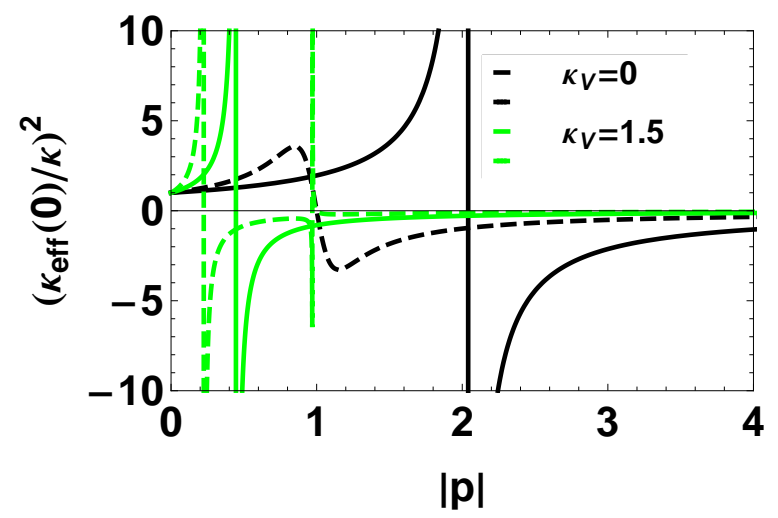

FIG. 11: The static effective screening length 79 versus polarization for different $\kappa_{V}$ and $B_{f}=0$ (solid) and $B_{f}=1$ (dashed).

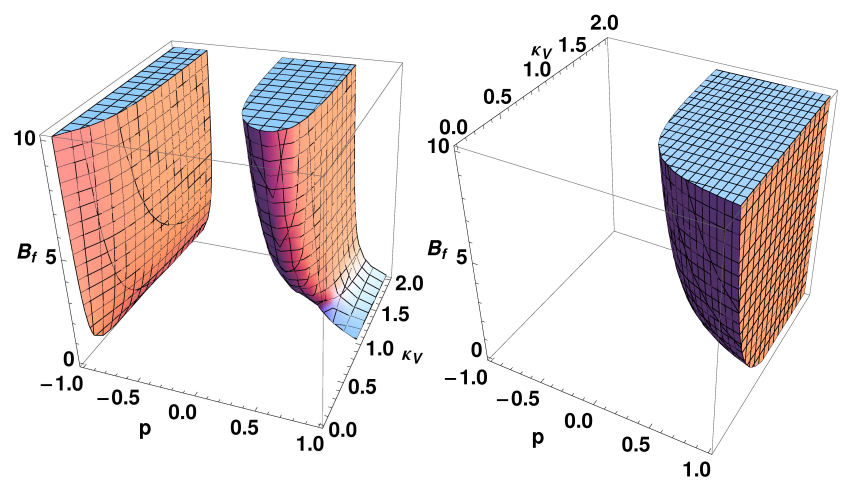

FIG. 12: (Color online) The range where the static effective screening length 79 becomes negative for $1 / \tau=1 \omega_{p}$ (left) and $1 / \tau=0 \omega_{p}$ (right).

the screening length which was treated other wise by extensive numerics 28183 .

The static screening length 79 changes only for finite $\kappa_{V}^{2}$ which means a finite magnetic field or ferromagnetic impurity polarization $V$. In other words we need a preferred direction of motion in order to see a change of static screening length. In the latter case it is then dependent on the spin-orbit coupling $B_{f}$ as illustrated in figure 11. One sees that the screening length increases with increasing polarization for $\kappa_{V}^{2} \neq 0$ and diverges at the zero of (79) which provides the critical $\kappa_{V}^{2}$ in terms of the polarization $p$ and the material parameter $\omega_{p} \tau$. This instability appears here in the spatial screening length and we can interpret it as a spatial domain separation of spinpolarized electrons known as domain wall formation 4 . Interestingly, for finite spin-orbit coupling there appears an upper singularity at $p=1$.

The range where the real part becomes negative is plotted in figure 12. With increasing collision frequency the range of instability becomes smaller.

Comparing the case with vanishing collision frequency in figure 12 we see that only one range at positive polar- 


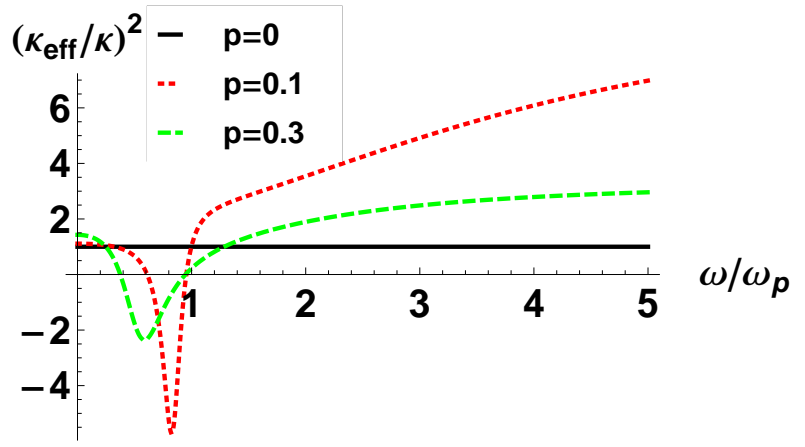

FIG. 13: The dynamical effective screening length 78 versus frequency for different polarizations, $\kappa_{V}=1,1 / \tau=0.3 \omega_{p}$ and $B_{f}=0$.

ization appears. In other words there appears an asymmetric second range in the instability due to the collisions for positive and negative polarizations.

It is interesting to discuss the dynamical screening length as well. First one notes that the correct static limit $\sqrt{79}$ only appears if we have a relaxation damping $1 / \tau$ which drops out of the result. In contrast if we first set $1 / \tau \rightarrow 0$ before the static limit we would obtain

$$
\lim _{\tau \rightarrow \infty}\left(\frac{\kappa^{\mathrm{eff}}(\omega)}{\kappa}\right)^{2}=\frac{\left(\omega^{2}-1\right)\left(\omega^{2}+p^{2}-1\right)}{\kappa_{V}^{2} p\left(\omega^{4}+p^{2}-1\right)}
$$

leading to the wrong static limit $-1 / p \kappa_{V}^{2}$ which is clearly unphysical. Therefore an even infinitesimal friction is necessary in order to ensure the correct static screening length. One can see this also from the limit of vanishing polarization $p \rightarrow 0$ which yields

$$
\lim _{p \rightarrow 0}\left(\frac{\kappa^{\mathrm{eff}}(\omega)}{\kappa}\right)^{2}=1-i \omega \tau
$$

The dynamical screening length is plotted in figures 13 and 14 for different cuts. Like in the static limit above, at certain $\kappa_{V}$ the dynamical screening length becomes negative indicating a domain-wall formation.

The range where the real part becomes negative is given in figure 15. With increasing collision frequency the range of instability becomes smaller. If we additionally demand that the imaginary part of the dynamical screening length should be positive which means spatially unstable modes, we get a smaller region. Above a certain collisional damping there is no such region.

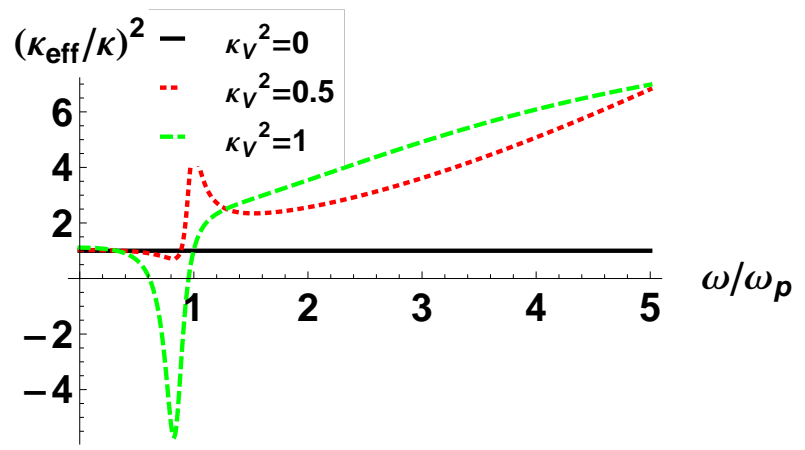

FIG. 14: The dynamical effective screening length 78 versus frequency for different $\kappa_{V}$, a polarization $p=0.1$, $1 / \tau=0.3 \omega_{p}$, and $B_{f}=0$.
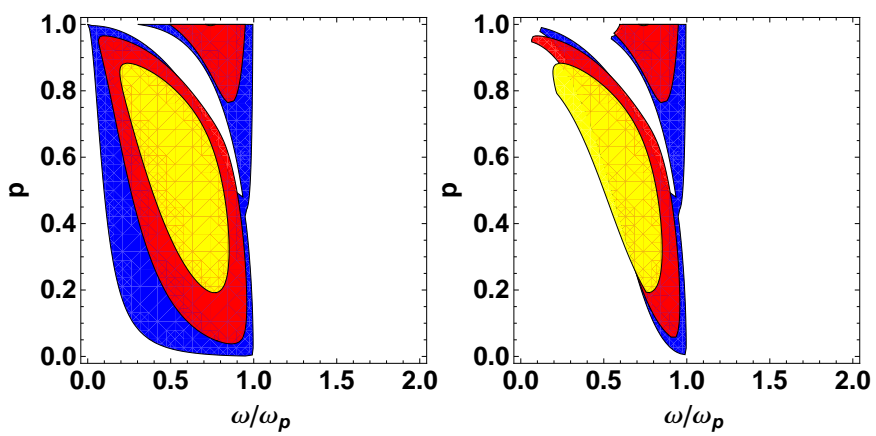

FIG. 15: (Color online) Left: The range where the dynamical effective screening length 78 becomes negative versus frequency and polarization for $\kappa_{V}=1$. The inner range (yellow) is for $1 / \tau=0.5 \omega_{p}$, the middle (red) for $1 / \tau=0.3 \omega_{p}$ and the outer (blue) one for $1 / \tau=0.1 \omega_{p}$. Right: additional demand that $\operatorname{Im} \kappa^{e f f}>0$

\section{Spin response}

The spin response $\delta \vec{s}=\vec{\chi}_{s} \Phi$ can be calculated as well and we obtain with $\vec{q} \Phi=i e \vec{E}_{q}$

$$
\frac{\delta \vec{s}_{q}}{E}=i \frac{e}{q} \vec{\chi}_{s}=\frac{e n}{\omega_{p}}\left(\begin{array}{c}
s_{1}(\omega) \frac{b_{1}(q)}{q}+B_{c} s_{2}(\omega) \frac{b_{2}(q)}{q} \\
s_{1}(\omega) \frac{b_{2}(q)}{q}-B_{c} s_{2}(\omega) \frac{b_{1}(q)}{q} \\
i \frac{q \epsilon_{0} \omega_{p}}{n e^{2}} s_{3}(\omega)
\end{array}\right)
$$

which means we have an induced spin due to an applied electric field as used in microwave spectroscopy 4 . This is purely transverse to the $z$-direction of the effective magnetic and ferromagnetization field for longwavelength. We consider this as the response of spinHall effect, described by the spin-orbit coupling $\vec{b}(q)=$ $\left(B_{\perp 1}, B_{\perp 2}, B_{\perp 3}\right)$ according to 56 and $(\mathrm{C} 6)$. Since $\vec{q}|| \vec{E}$ this q-dependent spin-orbit coupling describes the excitation due an external electric field. Oscillating electric currents are used experimentally to create an effective magnetic field and ferromagnetic resonances ${ }^{40}$.

The external magnetic field enters $(82)$ by the dimen- 
sionless quantity

$$
B_{c}=\frac{\omega_{c} \epsilon_{f}}{n D \hbar \omega_{p}^{2}}=\epsilon_{0} \frac{B}{n D e \hbar} \frac{\epsilon_{f}}{n} .
$$

The frequency-dependent functions 82 can be recast into the form

$$
\begin{aligned}
s_{1}+1 & =-\frac{1+p}{2} I(p)-\frac{1-p}{2} I(-p) \\
s_{2} & =\frac{2 I_{1}(p)}{1-p^{2}}-\frac{I(p)-I(-p)}{p}+\frac{I_{2}(p)}{p(1+p)}-\frac{I_{2}(-p)}{p(1-p)} \\
s_{3} & =-\frac{1+p}{2} I(p)+\frac{1-p}{2} I(-p)
\end{aligned}
$$

with

$$
\begin{array}{rlrl}
I(p) & =\frac{1}{-1-p+\frac{i}{\omega_{p}^{2} \tau} \omega+\frac{\omega^{2}}{\omega_{p}^{2}}} & \rightarrow-\omega_{p} \frac{\sin \gamma t}{\gamma} \mathrm{e}^{-\frac{t}{2 \tau}} \\
I_{1}(p)=\frac{1}{1-i \omega \tau} & \rightarrow \frac{1}{\tau} \mathrm{e}^{-\frac{t}{\tau}} \\
I_{2}(p)=\frac{i \omega}{\tau \omega_{p}^{2}} I_{1}(p) & \rightarrow \frac{1}{\tau} \frac{\partial}{\partial t} \frac{\sin \gamma t}{\gamma} \mathrm{e}^{-\frac{t}{2 \tau}}
\end{array}
$$

and

$$
\gamma=\sqrt{(1+p) \omega_{p}^{2}-\frac{1}{4 \tau^{2}}}
$$

Since we apply a frequency-constant electric field it means we have an instant disturbance of the system at time $t=0$ in the form $E(t)=E \delta(t)$. This field itself has to be subtracted from the response which is represented by the constant $s_{1}(\omega)+1$. Further we present the linearized result with respect to the spin-orbit coupling. A nonlinear analytic result with some more drastic simplifications can be found in $\frac{86}{}$.

The collisions are responsible for the damping of this oscillatory motion. Dependent on the temperature we will have a transition from collision-dominated damped motion towards an oscillatory regime as observed in 87 . This transition is here explicitly seen in the expression for $\gamma$ in (86) which turns the oscillatory behavior into an exponential one if

$$
(1+p) \omega_{p}^{2}<\frac{1}{4 \tau^{2}}
$$

which provides density, polarization, and (due to the relaxation time) temperature-dependent criteria for such a transition.

It is now interesting to inspect the spin response for linear Dresselhaus and Rashba spin-orbit coupling. If the electric field is excited in $x$-direction we have for Dresselhaus $b_{1}=\beta_{D} q_{x} / \Sigma_{n}, b_{2}=0$ and for Rashba $b_{1}=0, b_{2}=-\beta_{R} q_{x} / \Sigma_{n}$. This translates into the spin response

$$
\begin{aligned}
\left.\frac{\delta \vec{s}_{q}}{E}\right|_{D} & =\frac{\beta_{R} q_{x} e n}{q \Sigma_{n} \omega_{p}}\left(s_{1},-s_{2} B_{c}, 0\right) \\
\left.\frac{\delta \vec{s}_{q}}{E}\right|_{R} & =\frac{\beta_{D} q_{x} e n}{q \Sigma_{n} \omega_{p}}\left(-s_{2} B_{c},-s_{1}, 0\right)
\end{aligned}
$$

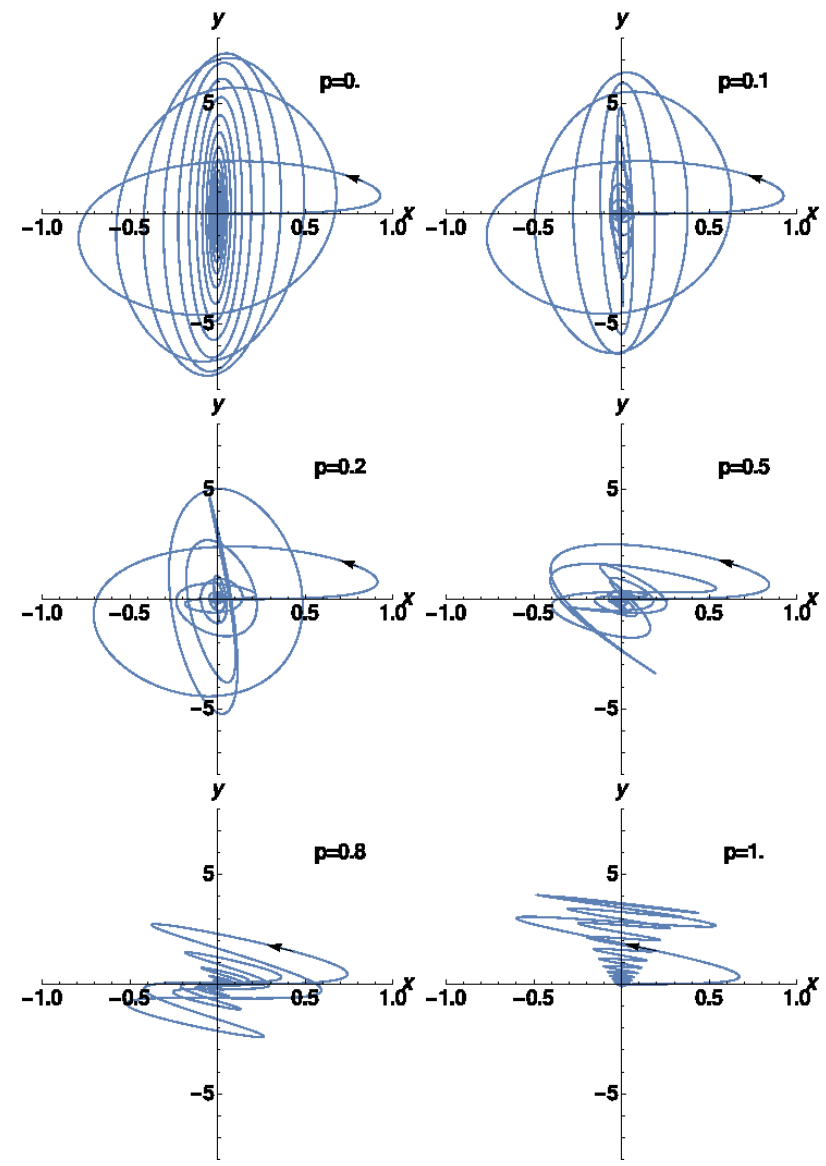

FIG. 16: (Movie online) The time-dependent trajectories of the induced spin with the disturbance of the electric field $\vec{E} \delta(t)$ in $x$-direction for Dresselhaus spin-orbit coupling and $1 / \tau=0.1 \omega_{p}$. The external magnetic field was chosen $B_{c}=1$ according to 83 .

If the electric field is excited in $y$-direction we have for Dresselhaus $b_{2}=-\beta_{D} q_{y} / q, b_{1}=0$ and for Rashba $b_{2}=0, b_{1}=\beta_{R} q_{y} / q$ and the response in (88) are interchanged between Dresselhaus and Rashba. Therefore it is sufficient to discuss one of the cases, say excitation in $x$-direction. Let us concentrate on the Dresselhaus relaxation. From 88 we see that the external magnetic field $B_{c}$ causes ellipsoid trajectories. If it is absent, we have a mere linear-polarized damped oscillation in x-direction.

In the next figure 16 we plot the trajectories for different polarizations.

One recognizes that with increasing polarization the spin response turns to the perpendicular direction of the applied electric field which is a spin-Hall effect. Here we can see how the evolution of trajectories changes with increasing polarization. The Rashba spin-orbit coupling will lead to the same curves but with $90^{\circ}$ clockwise rotation as one sees from 88 too.

Since the change of spins 82 is different in each spatial direction triggered by spin-orbit coupling and split further by the magnetic field one can predict that this will lead to an anomalous spin segregation as was ob- 

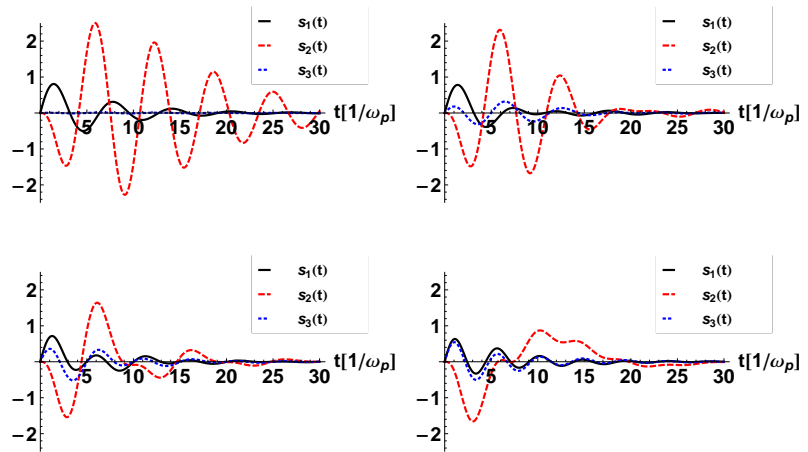

FIG. 17: The time dependent spin response function 82 for $1 / \tau=0.3 \omega_{p}$ and polarizations $p=0.01,0.3,0.6,0.9$ from upper left to lower right.

served in $\stackrel{88}{8}$ and investigated in one-dimensional systems in 89 .

The spin dephasing time is of special interest 90 where one has found discrepancies between the experimental values and earlier treatments. It is now quite difficult to extract dephasing times since the envelope of the oscillation in each direction shows maxima and a quite nonlinear behavior as illustrated in figure 17 by the constituent time-dependent functions of 82 . One sees that besides oscillations with the frequency (86) the $s_{1}(t)$ possesses a maximum and all functions become quite nonlinear for higher polarizations. These components mix additionally due to the spin-orbit coupling and the magnetic field. If we, nevertheless, fit these time dependence to a damped exponential oscillator, we can extract the spin-dephasing time $\tau_{s}$ analogously to 2 .

The results are given in figure 18 . The overall observation is that the spin dephasing time is an order of magnitude larger than the relaxation time. One sees that the $s_{1}$ component, which corresponds to the $x$-component for the Dresselhaus and $y$ component for the Rashba coupling has a minimum at a polarization which increases with increasing relaxation time. The minima in $s_{2}$ and $s_{3}$ are not so pronounced and shift to larger polarizations as well with increasing relaxation time. This result is different from ${ }^{92}$ where only an increasing spin dephasing time in dependence on the polarization has been reported. The combined effect of the Rashba and the Dresselhaus coupling as well as the magnetic field mixes these results according to 82 .

Here, we extracted the spin-dephasing time as an envelope of the precessional motion of the spins after a sudden distortion by an electric field. This is the Dyakonov-Perel mechanism of relaxation, e.g. investigated experimentally and theoretically in $56+58$
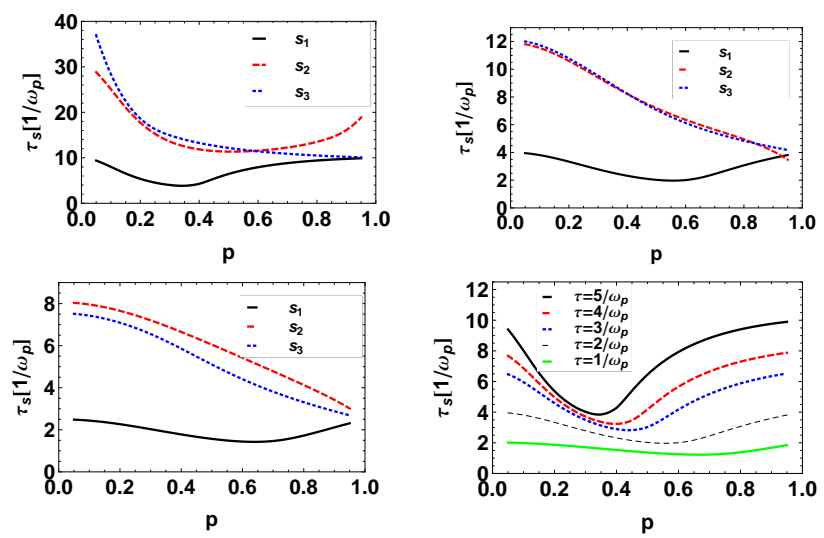

FIG. 18: The spin dephasing times 82 from exponential envelops for the different directions versus polarization with $1 / \tau=0.2,0.5,0.8$ from upper left to lower left and $s_{1}$ for different relaxation times (lower right).

\section{RESPONSE WITH MAGNETIC FIELDS}

\section{A. Linearizing kinetic equation with magnetic field}

We want to consider the spin and density response to an external perturbing electric field now under a constant bias of magnetic field. The magnetic field consists of a constant and an induced part $B(x, t)=B+\delta B(x, t)$. Since the external electric field perturbation is produced due to an external potential $U^{e x t}$ one sees from the Maxwell equation that $\delta \dot{\vec{B}}=-\nabla \times \delta \vec{E}=0$, which means that all terms linear in the induced magnetic field vanish. It is convenient to work in velocity variables instead of momentum defined according to (4) and we use for the quasiparticle energy $\epsilon_{p}=p^{2} / 2 m_{e}+\Sigma_{0}$. We obtain finally from 3 with the Larmor frequency $\omega_{c}=\frac{e B}{m_{e}}$

$$
\begin{aligned}
& {\left[-i \omega+i q v+\frac{1}{\tau}+\left(v \times \omega_{c}\right) \partial_{v}\right] \delta f} \\
& +\left[\frac{i q}{m_{e}} \partial_{v} \Sigma_{i}+\left(\vec{\tau}^{-1}\right)_{i}-\left(\frac{\partial_{v} \Sigma_{i}}{m_{e}} \times \omega_{c}\right) \partial_{v}\right] \delta g_{i}=S_{0}
\end{aligned}
$$

and

$$
\begin{aligned}
& {\left[-i \omega+i q v+\frac{1}{\tau}+\left(v \times \omega_{c}\right) \partial_{v}\right] \delta g_{i}-2 m_{e}(\Sigma \times \delta g)_{i}} \\
& +\left[\frac{i q}{m_{e}} \partial_{v} \Sigma_{i}+\left(\vec{\tau}^{-1}\right)_{i}-\left(\frac{\partial_{v} \Sigma_{i}}{m_{e}} \times \omega_{c}\right) \partial_{v}\right] \delta f=S_{i}
\end{aligned}
$$


with the source terms arising from the external field $\vec{q} \Phi=$ $i e \vec{E}$ and the induced meanfield variations 37 .

$$
\begin{aligned}
& S_{0}=\frac{i q \partial_{v} f}{m_{e}} \Phi+\frac{\delta n}{\tau \partial_{\mu} n} \partial_{\mu} f_{0} \\
& +\left(i \frac{q}{m_{e}}+\omega_{c} \times \frac{\partial_{v}^{\Sigma}}{m_{e}}\right) \delta \Sigma_{0} \partial_{v} f+\left(i \frac{q}{m_{e}}-\omega_{c} \times \frac{\partial_{v}^{\Sigma}}{m_{e}}\right) \delta \Sigma_{i} \partial_{v} g_{i} \\
& S_{i}=\frac{i q \partial_{v} g_{i}}{m_{e}} \Phi+2(\delta \Sigma \times g)_{i}+\frac{\delta n \partial_{\mu} g}{\tau \partial_{\mu} n} e_{i} \\
& +\left(i \frac{q}{m_{e}}+\omega_{c} \times \frac{\partial_{v}^{\Sigma}}{m_{e}}\right) \delta \Sigma_{0} \partial_{v} g_{i}+\left(i \frac{q}{m_{e}}-\omega_{c} \times \frac{\partial_{v}^{\Sigma}}{m_{e}}\right) \delta \Sigma_{i} \partial_{v} f .
\end{aligned}
$$

Compared with the result without magnetic field we see that the source terms 200 get additional rotation terms coupled to the momentum-dependent derivation of meanfields (37) which is present only with extrinsic spinorbit coupling. Further the drift side gets an explicit derivative with respect to the velocity which we will take into account in the following.

\section{B. Solution of linearized equations}

In order to solve 89 and 90 we use the same coordinate system as Bernstein ${ }^{93}$. The magnetic field $\vec{B}$ points in the $v_{z}$ direction and the $q$ vector is in the $v_{z}-v_{x}$ plane with an angle $\Theta$ between $v_{x}$ and $q$

$$
\vec{q}=q \sin \Theta \vec{e}_{x}+q \cos \Theta \vec{e}_{z} .
$$

For the velocity $v$ we use polar coordinates around $\vec{B}$ with an azimuthal angle $\phi$

$$
\vec{v}(\phi)=w \cos \phi \vec{e}_{x}+w \sin \phi \vec{e}_{y}+u \vec{e}_{z}
$$

and one gets

$$
\begin{aligned}
& \frac{1}{\tau}-i \omega+i \vec{q} \vec{v}+\left(\vec{v} \times \frac{e \vec{B}}{m}\right) \overrightarrow{\partial_{v}}=\frac{1}{\tau}-i \omega+i \vec{q} \cdot \vec{v}(\phi)-\omega_{c} \partial_{\phi} \\
& =\frac{1}{\tau}-i \omega+i \vec{q} \cdot \vec{v}\left(\omega_{c} t_{\phi}\right)-\partial_{t} \equiv-i \Omega_{t_{\phi}}-\partial_{t_{\phi}}
\end{aligned}
$$

with the orbiting time

$$
t_{\phi}=\phi / \omega_{c} .
$$

We can write the equations 89 and 90 as

$$
\begin{aligned}
& \left(-i \Omega_{t_{\phi}}-\partial_{t_{\phi}}\right) \delta f+\left(\frac{i q \partial_{v} \vec{\Sigma}}{m_{e}}+\vec{\tau}^{-1}\right) \cdot \vec{\delta} g=S_{0} \\
& \left(-i \Omega_{t_{\phi}}-\partial_{t_{\phi}}\right) \delta g_{i}+\left(\frac{i q \partial_{v} \Sigma_{i}}{m_{e}}+\left(\vec{\tau}^{-1}\right)_{i}\right) \delta f-2(\vec{\Sigma} \times \vec{\delta} g)_{i}=S_{i} .
\end{aligned}
$$

where the corresponding right hand sides are given by (91). Now we employ the identity

$$
\begin{aligned}
& \overrightarrow{\mathcal{B}} \cdot \delta \vec{g}+(\vec{\sigma} \cdot \overrightarrow{\mathcal{B}}) \delta f-2 \vec{\sigma} \cdot(\vec{\Sigma} \times \delta \vec{g})= \\
& \vec{\sigma} \cdot \frac{\overrightarrow{\mathcal{B}}+2 i \vec{\Sigma}}{2} \delta \hat{F}+\delta \hat{F} \vec{\sigma} \cdot \frac{\overrightarrow{\mathcal{B}}-2 i \vec{\Sigma}}{2}
\end{aligned}
$$

with $\overrightarrow{\mathcal{B}}=i q \partial_{v} \vec{\Sigma} / m_{e}+\vec{\tau}^{-1}$ and $\delta \hat{F}=\delta f+\vec{\sigma} \cdot \delta \vec{g}$ which one proves with the help of $(\tau a)(\tau b)=a \cdot b+i \tau(a \times b)$. This allows to rewrite 96$)$ into

$$
\begin{aligned}
& -\partial_{t_{\phi}} \hat{\delta} F-i \Omega_{t_{\phi}} \hat{\delta} F \\
& +\vec{\sigma} \cdot\left(\frac{\overrightarrow{\mathcal{B}}}{2}+i \vec{\Sigma}\right) \delta \hat{F}+\delta \hat{F} \vec{\sigma} \cdot\left(\frac{\overrightarrow{\mathcal{B}}}{2}-i \vec{\Sigma}\right)=\hat{S}_{p_{\phi}}(\omega)
\end{aligned}
$$

where $\hat{S}_{p_{\phi}}=S_{0}+\vec{\sigma} \cdot \vec{S}$.

Please note that due to (95) the integration over the azimuthal angle is translated into the time integration about orbiting intervals. Therefore Eq. $\sqrt{98}$ has a great similarity to the time-dependent Eq. (32).

Equation (98) is easily solved as

$$
\begin{aligned}
& \delta F=-\int_{\infty}^{t} d \bar{t} \mathrm{e}^{i \int_{t}^{\bar{t}} \Omega_{t^{\prime}}^{+} d t^{\prime}} \mathrm{e}^{\vec{\sigma} \int_{t}^{t}\left(\frac{\overrightarrow{\mathcal{B}}_{t^{\prime}}}{2}+i \vec{\Sigma}_{t^{\prime}}\right) d t^{\prime}} \hat{S}_{p_{\omega_{c} \bar{t}}} \mathrm{e}^{\vec{\sigma} \int_{t}^{t}\left(\frac{\overrightarrow{\mathcal{B}}_{t^{\prime}}}{2}-i \vec{\Sigma}_{t^{\prime}}\right) d t^{\prime}} \\
& =\int_{-\infty}^{0} d x \mathrm{e}^{-i \int_{0}^{x} \Omega_{t-y}^{+} d y} \\
& \times \mathrm{e}^{\vec{\sigma} \int_{0}^{x}\left(\frac{1}{2} \overrightarrow{\mathcal{B}}_{t-y}+i \vec{\Sigma}_{t-y}\right) d y} \hat{S}_{p_{\omega_{c}(t-x)}}(\omega) \mathrm{e}^{\vec{\sigma} \int_{0}^{x}\left(\frac{1}{2} \overrightarrow{\mathcal{B}}_{t-y}-i \vec{\Sigma}_{t-y}\right) d y}
\end{aligned}
$$

where we used $\omega^{+}=\omega+i \tau^{-1}$ as before. The first exponent can be calculated explicitly with the definitions of (93) and 94

$$
i \int_{0}^{x} \Omega_{t-y}^{+} d y=i \omega^{+} x-i \vec{q} \cdot \mathcal{R}_{x} \cdot \vec{v}(t)
$$

with the matrix ${ }^{94}$

$$
\mathcal{R}_{x}=\frac{1}{\omega_{c}}\left(\begin{array}{ccc}
\sin \omega_{c} x & 1-\cos \omega_{c} x & 0 \\
\cos \omega_{c} x-1 & \sin \omega_{c} x & 0 \\
0 & 0 & \omega_{c} x
\end{array}\right)
$$

having the property $\mathcal{R}_{-x}=-\mathcal{R}_{x}^{T}$. Neglecting the magnetic-field dependence in the phase $q R v=q v+o(B)$ we obtain with (99) exactly again the solution (34) but with an additional retardation in the momentum $S_{p_{\omega_{c}(t-x)}}$ instead of $S_{p_{\omega_{c} t}}=S_{p}$ in (34).

We employ the long-wavelength approximation $\overrightarrow{\mathcal{B}} \approx 0$ neglecting the vector relaxation. The integration over an azimuthal angle $x=\phi / \omega_{c}$ is coupled to the momentum (velocity) arguments. The spin-orbit coupling provides a momentum-dependent $\vec{\Sigma}$ which couples basically to $q \partial_{p} \vec{\Sigma}$. Since

$$
m_{e} q \partial_{p}=q \sin \Theta\left(\cos \phi \partial_{w}-\frac{\sin \phi}{w} \partial_{\phi}\right)+q \cos \Theta \partial_{u}
$$


in the coordinates 92 and 93, it means we neglect higher than first order derivatives in $\phi$ and $\partial_{p} \vec{\Sigma}$ when approximating $\vec{\Sigma}_{t-y} \approx \vec{\Sigma}_{t}$ in the exponent. We obtain $\delta \hat{F}=\delta f+\vec{\sigma} \cdot \delta \vec{g}=\int_{-\infty}^{0} d x \mathrm{e}^{i\left(\vec{q} \mathcal{R}_{x} \vec{v}_{t}-\omega^{+} x\right)} \mathrm{e}^{i x \vec{\sigma} \vec{\Sigma}_{t}} \hat{S}_{p_{\omega_{c}(t-x)}} \mathrm{e}^{-i x \vec{\sigma} \vec{\Sigma}_{t}}$.

To work it out further we use $\mathrm{e}^{i \tau \cdot a}=\cos |a|+i \frac{\tau \cdot a}{|a|} \sin |a|$ to see that

$$
\begin{aligned}
& \mathrm{e}^{i \vec{\sigma} \cdot \vec{\Sigma} x}\left(S_{0}+\vec{\sigma} \cdot \vec{S}\right) \mathrm{e}^{-i \vec{\sigma} \cdot \vec{\Sigma} x}=S_{0}+(\vec{\sigma} \cdot \vec{S}) \cos (2 x|\Sigma|) \\
& +\vec{\sigma}(\vec{S} \times \vec{e}) \sin (2 x|\Sigma|)+(\vec{\sigma} \cdot \vec{e})(\vec{S} \cdot \vec{e})(1-\cos (2 x|\Sigma|))
\end{aligned}
$$

with the direction $\vec{e}=\vec{\Sigma} /|\Sigma|$ and $(8)$.

The effect of a magnetic field is basically condensed at two places. First the phase term $\vec{q} \cdot \mathcal{R}_{x} \cdot \vec{p}=\vec{q} \cdot \vec{p}+o(B)$ and we have $\bar{\omega}=\omega-\vec{p} \cdot \vec{q} / m+i / \tau$

$$
\begin{aligned}
& \int_{-\infty}^{0} \mathrm{e}^{-i\left(\omega x-\vec{q} \cdot \mathcal{R}_{x} \cdot \frac{\vec{p}}{m}\right)}\left(\begin{array}{c}
\cos 2|\Sigma| x \\
1-\cos 2|\Sigma| x \\
-\sin 2|\Sigma| x
\end{array}\right) \\
& =\frac{1}{2}\left(\begin{array}{c}
\frac{i}{\overline{\bar{\omega}+2 \Sigma}}+\frac{i}{\bar{\omega}-2 \Sigma} \\
\frac{2 i}{\bar{\omega}}-\frac{i}{\bar{\omega}+2 \Sigma}-\frac{i}{\bar{\omega}-2 \Sigma} \\
\frac{1}{\bar{\omega}-2 \Sigma}-\frac{1}{\bar{\omega}+2 \Sigma}
\end{array}\right)+o\left(q^{2}, B\right)=\left(\begin{array}{c}
\frac{i}{\bar{\omega}} \\
0 \\
\frac{2 \Sigma}{\omega^{2}}
\end{array}\right)+o\left(B, \Sigma^{2}\right) .
\end{aligned}
$$

The magnetic-field-dependent phase factor $\mathcal{R}_{x}$ does play a role only in inhomogeneous systems with finite wavelength. In the limit of large wavelength this effect can be ignored.

The sin and cos terms are results of the precession of spins around the effective direction $\vec{e}=\vec{\Sigma} / \Sigma$ and can be considered as Rabi oscillations. For the limit of small $\Sigma$ we can expand the $\cos$ and $\sin$ terms in first order $\approx S_{0}+\vec{\sigma} \cdot \vec{S}-2 \vec{\sigma} \cdot(\vec{S} \times \vec{\Sigma}) x$ as was analyzed in 95 . The second effect is the retardation in $t=\phi / \omega_{c}$ which means that the precession time in the arguments $S(t-x)$ contains important magnetic field effects. In fact, this retardation represents all kinds of normal Hall effects as we will convince ourselves now.

\section{Retardation subtleties by magnetic field}

The magnetic field causes a retarding integral in the last section over the precession time $t=\phi / \omega_{c}$ coupled to any momentum by the representation in Bernstein coordinates

$$
\frac{\vec{p}_{\phi}}{m}=(w \cos \phi, w \sin \phi, u) .
$$

This retardation is crucial for any kind of Hall effect. In order to get a handle on such expressions we concentrate first on the mean values of the scalar part $\delta f$. The general field-dependent solution provides a form

$$
\langle A\rangle=\sum_{p_{\phi}} \int_{-\infty}^{0} d x \mathrm{e}^{-i\left(\omega^{+} x-\vec{q} \mathcal{R}_{x} \frac{\vec{p}_{\phi}}{m}\right)} A\left(\vec{p}_{\phi}\right) S_{0}\left(p_{\phi-x \omega_{c}}\right)(
$$

where $S_{0}$ is the scalar source term. The trick is to perform first a shift $\phi \rightarrow \phi+\omega_{c} x$ and integrate then about $p=p_{\phi}$. This has the effect that the retardation is only condensed in the momentum of variable $A$

$$
\begin{aligned}
& \mathcal{P}(x)=\vec{p}_{\phi+\omega_{c} x} \\
& =\vec{p}_{\phi} \cos \left(\omega_{c} x\right)+\vec{e}_{z}\left(\vec{e}_{z} \cdot \vec{p}_{\phi}\right)\left[1-\cos \left(\omega_{c} x\right)\right]+\vec{e}_{z} \times \vec{p}_{\phi} \sin \left(\omega_{c} x\right) \\
& =\vec{p}_{\phi}+\vec{e}_{z} \times \vec{p}_{\phi} \omega_{c} x+o\left(\omega_{c}^{2}\right) .
\end{aligned}
$$

and the exponent

$$
\begin{aligned}
\mathcal{R}_{x} \frac{\vec{p}_{\phi+\omega_{c} x}}{m} & =\frac{\vec{p}_{\phi}}{m} \frac{\sin \omega_{c} x}{\omega_{c}}+\left(\vec{e}_{z} \times \frac{\vec{p}_{\phi}}{m}\right) \frac{1-\cos \omega_{c} x}{\omega_{c}} \\
& =\frac{\vec{p}_{\phi}}{m} x+\omega_{c} \frac{x^{2}}{2} \vec{e}_{z} \times \frac{\vec{p}_{\phi}}{m}+o\left(\omega_{c}^{2}\right) .
\end{aligned}
$$

The phase effect leads to the first order corrections in $\omega_{c}$ or alternatively in wave length $q$

$$
\begin{aligned}
& \langle A\rangle=\sum_{p} S_{0}(p)\left[1-\frac{\omega_{c}}{2 m} \vec{q} \cdot\left(\vec{e}_{z} \times \vec{p}\right) \partial_{\omega}^{2}+o\left(\omega_{c}^{2}\right)\right] \\
& \times \int_{-\infty}^{0} d x \mathrm{e}^{-i\left(\omega^{+} x-\frac{\vec{q} \cdot \vec{p}}{m}\right)} A[\mathcal{P}(x)] \\
& =\sum_{p} S_{0}(p) A\left[\mathcal{P}\left(i \partial_{\omega}\right)\right] \\
& \times\left[1-\frac{\omega_{c}}{2 m} \vec{q} \cdot\left(\vec{e}_{z} \times \vec{p}\right) \partial_{\omega}^{2}+o\left(\omega_{c}^{2}\right)\right] \frac{i}{\omega^{+}-\frac{\vec{q} \cdot \vec{p}}{m}} .
\end{aligned}
$$

where the integration variable $x$ in the momentum (108) can be transformed into derivatives of $\omega$ if needed.

Completely analogously we can perform any mean value over the vector part of the distribution $\vec{\delta} g$. We have

$$
\begin{aligned}
& \langle\vec{A}\rangle=\sum_{p} A(p) \delta \vec{g}=\sum_{p}\left[1-\frac{\omega_{c}}{2 m} \vec{q} \cdot\left(\vec{e}_{z} \times \vec{p}\right) \partial_{\omega}^{2}+o\left(\omega_{c}^{2}\right)\right] \\
& \times \int_{-\infty}^{0} d x \mathrm{e}^{i\left(\frac{\vec{q} \vec{p}}{m} x-\omega^{+} x+o\left(\omega_{c}, q^{2}\right)\right)} A[\mathcal{P}(x)] \\
& \times[\vec{S} \cos (2 \Sigma x)+\vec{e} \times \vec{S} \sin (2 \Sigma x)+\vec{e}(\vec{e} \cdot \vec{S})(1-\cos (2 \Sigma x))]
\end{aligned}
$$

where the arguments of $\vec{S}, \Sigma$ and $\vec{e}$ are the momentum $p$ and no retardation anymore. The exponent can be written in complete B-dependence with $\mathcal{R}_{x}$ of course. Then the $\mathrm{x}$-integration over the $\cos$ and $\sin$ terms has to be performed numerically. Analytically we can proceed if 
we expand the phase effect in orders of $\vec{q}$. We obtain with the help of 105)

$$
\begin{aligned}
& \langle\vec{A}\rangle=\sum_{p} A\left[\mathcal{P}\left(i \partial_{\omega}\right)\right]\left[1-\frac{\omega_{c}}{2 m} \vec{q} \cdot\left(\vec{e}_{z} \times \vec{p}\right) \partial_{\omega}^{2}+o\left(\omega_{c}^{2}\right)\right] \\
& \times\left[[\vec{e} \times(\vec{S} \times \vec{e})] \frac{i}{2}\left(\frac{1}{\bar{\omega}+2 \Sigma}+\frac{1}{\bar{\omega}-2 \Sigma}\right)\right. \\
& \left.+\vec{e} \times \vec{S} \frac{1}{2}\left(\frac{1}{\bar{\omega}+2 \Sigma}-\frac{1}{\bar{\omega}-2 \Sigma}\right)+\vec{e}(\vec{e} \cdot \vec{S}) \frac{i}{\bar{\omega}}\right]
\end{aligned}
$$

with $\bar{\omega}=\omega+\frac{i}{\tau}-\frac{\vec{p} \vec{q}}{m}$ and 108 .

The formula (110) and (112) establish the rules for calculating mean values with magnetic fields. The usefulness of these rules can be demonstrated since it simplifies the way to obtain the linearized solutions 40, 41) and (43) tremendously. In fact integrating with $A=1$ we obtain straightforwardly the response functions and the equation system (46). This shows that up to linear order in wave-vector the magnetic field enters only via the Zeeman term in $\vec{\Sigma}$.

\section{Classical Hall effect}

Now, we are in a position to see how the Hall effect is buried in the theory. Therefore we neglect any meanfield and spin-orbit coupling for the moment such that the $f$ and $\mathrm{g}$ distributions decouple and use the $q \rightarrow 0$ limit, i.e. homogeneous situation. We obtain from 99 with (104) and (91)

$$
\delta f=-\int_{-\infty}^{0} d x \mathrm{e}^{-i \omega^{+} x} e \vec{E} \cdot \overrightarrow{\partial_{p_{\phi}}} f\left(p_{\phi-\omega_{c} x}\right)
$$

where we now pay special care to the retardation since this provides the Hall effect which was overseen in many treatments of magnetized plasmas.

After the shift of coordinates in azimuthal angle $\phi$ as outlined in the last section, we can carry out the $x$ integration with the help of (108), 1110) and (105):

$$
\begin{aligned}
\vec{J}= & e \sum_{p_{\phi}} \frac{\overrightarrow{p_{\phi}}}{m_{e}} \delta f \\
= & -e^{2} \sum_{p_{\phi}} \vec{E} \cdot \overrightarrow{\partial_{p_{\phi}}} f\left(p_{\phi}\right) \int_{-\infty}^{0} d x \mathrm{e}^{-i \omega^{+} x} \frac{\vec{p}_{\phi+\omega_{c} x}}{m} \\
= & \sigma_{0} \frac{1-i \omega \tau}{(1-i \omega \tau)^{2}+\left(\omega_{c} \tau\right)^{2}}\left[\vec{E}+\frac{\left(\omega_{c} \tau\right)^{2}}{(1-i \omega \tau)^{2}}\left(\vec{E} \cdot \vec{e}_{z}\right) \vec{e}_{z}\right. \\
& \left.+\frac{\omega_{c} \tau}{1-i \omega \tau} \vec{E} \times \vec{e}_{z}\right]
\end{aligned}
$$

which agrees of course with the elementary solution of

$$
m_{e} \dot{\vec{v}}=e(\vec{v} \times \vec{B})+e \vec{E}-m_{e} \frac{\vec{v}}{\tau} .
$$

In order to obtain all three precession terms we have used the complete form 108 and no expansion in $\omega_{c}$.

\section{E. Quantum Hall effect}

If we consider low temperatures such that the motion of electrons become quantized in Landau levels we have to use the quantum kinetic equation and not the quasi-classical one. However, we can establish a simple re-quantization rule which allows us to translate the above discussed quasi-classical results into the quantum expressions. Therefore we recall the linearization of the quantum-Vlasov equation, which is the quantum kinetic equation with only the mea-field in operator form:

$$
\dot{\rho}-\frac{i}{\hbar}[\rho, H]=0 .
$$

The perturbing Hamiltonian due to external electric fields is $\delta H=e \vec{E} \cdot \overrightarrow{\hat{x}}$ such that the linearization in eigenstates $E_{n}$ of the unperturbed Hamiltonian reads

$$
\delta \rho_{n n^{\prime}}=-e \vec{E} \cdot \vec{x}_{n n^{\prime}} \frac{\rho_{n}-\rho_{n^{\prime}}}{\hbar \omega-E_{n}+E_{n^{\prime}}} .
$$

One obtains the same result in the vector gauge since $[\rho, \delta H]=\frac{e \vec{E} t}{m_{e}}[\rho, \vec{p}]=e \vec{E}[\rho, \vec{v}]$ and the same matrix elements appear. Now, we investigate the quasi-classical limit where the momentum states are proper representations. We chose

$$
\left\langle n\left|=\left\langle p_{1}\left|=\left\langle p+\frac{q}{2}|, \quad| n\right\rangle^{\prime}=\right| p_{2}\right\rangle=\right|-p+\frac{q}{2}\right\rangle
$$

and we have in the quasi-classical $q \rightarrow 0$ approximation

$$
\begin{aligned}
\vec{x}_{n n^{\prime}}\left(\rho_{n}-\rho_{n^{\prime}}\right) & =\frac{\hbar}{i} \overrightarrow{\partial_{q}} \delta(\vec{q})\left(\rho_{p+\frac{q}{2}}-\rho_{p-\frac{q}{2}}\right) \\
& \approx \frac{\hbar}{i} \overrightarrow{\partial_{q}} \delta(\vec{q}) \vec{q} \cdot \overrightarrow{\partial_{p}} \rho_{p}=-\frac{\hbar}{i} \delta(\vec{q}) \overrightarrow{\partial_{p}} \rho_{p}
\end{aligned}
$$

from which follows

$$
\delta \rho \approx-i \hbar \frac{e \vec{E} \cdot \overrightarrow{\partial_{p}} \rho}{\hbar \omega-\frac{\vec{p} \cdot \vec{q}}{m_{e}}} .
$$

This is precisely the quasi-classical result we obtain from quasi-classical kinetic equations. Turning the argument around we see that we can re-quantize our quasi-classical results by applying the rule

$$
\vec{E} \cdot \overrightarrow{\partial_{p}} f \rightarrow \vec{E} \cdot \vec{v}_{n n^{\prime}} \frac{f_{n}-f_{n^{\prime}}}{E_{n^{\prime}}-E_{n}}
$$

Let us apply it to the normal Hall conductivity. We use the area density $1 / A$ and re-normalize the level distribution $\sum_{n} f_{n}=1$ to obtain for the static conductivity $\omega=0$

$$
\sigma_{\alpha \beta}=\frac{e^{2} \hbar i}{A} \sum_{n n^{\prime}} f_{n}\left(1-f_{n^{\prime}}\right) \frac{1-\mathrm{e}^{\beta\left(E_{n}-E_{n^{\prime}}\right)}}{\left(E_{n}-E_{n^{\prime}}\right)^{2}} v_{n n^{\prime}}^{\alpha} v_{n^{\prime} n}^{\beta}
$$

which is nothing but the Kubo formula. Further evaluation for Landau levels has been performed by 
Vasilopoulos ${ }^{96 \mid 97}$. Therefore one chose the gauge $\vec{A}=$ $(0, B x, 0)$ and the corresponding energy levels are

$$
E_{n}=\left(n+\frac{1}{2}\right) \hbar \omega_{c}+\frac{p_{z}^{2}}{2 m_{e}}
$$

where the last term is only in 3D. The wave functions read

$$
|n\rangle=\frac{1}{\sqrt{A}} \phi_{n}\left(x+x_{0}\right) \mathrm{e}^{i p_{y} y / \hbar} \mathrm{e}^{i p_{z} z / \hbar}
$$

with the harmonic oscillator functions $\phi_{n}, x_{0}=l^{2} p_{y} / \hbar$, $l^{2}=\hbar / e B$, and $A=L_{y} L_{z}$ where the corresponding $z$ parts are absent in $2 \mathrm{D}$. The calculation in $3 \mathrm{D}$ can be found in $[97$. Here, we represent the $2 \mathrm{D}$ calculation. One easily obtains

$$
v_{n n^{\prime}} v_{n^{\prime} n}=\frac{i \hbar \omega_{c}}{2 m}\left[n \delta_{n^{\prime}, n-1}-(n+1) \delta_{n^{\prime}, n+1}\right] \delta\left(p_{y}-p_{y}^{\prime}\right) .
$$

Introducing this into 122 and using

$$
\sum_{p_{y}}=\frac{L_{y}}{2 \pi \hbar} \int_{-\frac{\hbar L_{x}}{2 l^{2}}}^{\frac{\hbar L_{x}}{2 l^{2}}} d p_{y}=\frac{A}{2 \pi l^{2}}
$$

one arrives at

$$
\frac{e^{2}}{h} \sum_{n^{\prime}}\left(n^{\prime}+1\right) f_{n^{\prime}}\left(1-f_{n^{\prime}+1}\right)\left(1-\mathrm{e}^{-\beta \hbar \omega_{c}}\right) \rightarrow \frac{e^{2}}{h}(\bar{n}+1)
$$

with $\bar{n} \omega_{c} \leq \epsilon_{f} \leq(\bar{n}+1) \omega_{c}$. This is von Klitzing's result for $T \rightarrow 0$.

\section{F. Polarization functions}

Integrating 99 over the momentum $p=v m_{e}$ and solving algebraically for $\delta n$ and $\delta s$ one gets the response functions (46) with the B-field modifications. This concerns the precession-time integration instead of the energy denominator coupled to the tensor $q \mathcal{R} p$ and retardations in the momentum integration as described above. Especially with the help of 1112 the discussed polarizations (47)-(52) can be easily translated with (105) such that the effect of the magnetic field in the phase can be considered. The retardations does not play any role since for the density and spin response we do not have moments of momentum that would be retarded. The numerical results of these phase effects for small $\Sigma$ have been discussed in $\frac{95}{}$ leading to a staircase structure of the response functions with respect to the frequency at Landau levels. The excitation shows a splitting of the collective mode into Bernstein modes. Since it was presented in ${ }^{95}$ the repetition of results is avoided here.

\section{SUMMARY}

We have solved the linearized coupled kinetic equations for the density and spin Wigner distributions in arbitrary magnetic fields, vector and scalar meanfields, spin-orbit coupling and relaxation time approximations obeying the conservation of density. The response functions for the density and spin polarization with respect to an external electric field are derived. Various forms of polarization functions appear reflecting the complicated nature of different precessions and including Rabi shifts due to the effective Zeeman field. The latter consists of the magnetic field, the magnetization due to impurities, the spin polarization, and the spin-orbit coupling.

The long wavelength expansions are presented and the density and spin collective modes are determined for neutral and charged scattering separately. For a neutral system, no optical charge mode appears but there are three optical spin modes. These are dependent on the spin-orbit coupling and the effective Zeeman field. The energy and damping of these modes are found to be linearly dependent on the spin-orbit coupling. A spin-wave instability is reported and the range where such spin segregation can appear are calculated.

The charge and spin waves for charged Coulomb scattering show that only transverse spin modes can exist with respect to an effective magnetization axis. The charge density waves are damped plasma oscillations and the spin waves are splitting into two modes dependent on the polarization. One mode decreases in energy and becomes damped with increasing polarization while the second mode increases and becomes sharper again with increasing polarization. This analysis was possible with the help of the polarization, magnetic field and spin-orbit dependent dielectric function which was presented here as a new result. The range of instability with respect to frequency, polarization and collisional damping is presented which is again interpreted as spin segregation. The latter view is supported by the discussion of the statical and dynamical screening length whose dependence on the polarization and spin-orbit coupling is derived.

Finally, the spin response shows an interesting damped oscillation behavior different in each direction originating from the off-diagonal responses. The magnetic field causes an ellipsoidal relaxation which shows a rotation of the polarization axes depending on the spin-orbit coupling. We find a cross-over from damped oscillation to exponentially decay dependent on the polarization and collision frequency. Spin segregation as a consequence is discussed and the dephasing times are extracted.

The response with an external magnetic field shows some subtleties in retardations when observables of the Wigner functions are calculated. In fact, the Hall effect is possible to obtain only when these retardations are taken into account. The quantum version of the quasi-classical kinetic equations is shown to provide the quantum-Hall effect. Explicit calculations of the response function show a staircase behavior with respect to the frequency at the 
Landau levels. At these frequencies the Rabi satellite response functions become large leading to out-of-plane resonances 95 .

\section{Appendix A: Solving vector equations}

In the following all symbols are vectors and we search for solutions $y$ in terms of capital symbols. We start with the simplest vector equation

$$
y_{1}=B-Q\left(V \cdot y_{1}\right)
$$

which is easily solved by iteration and the geometrical sum

$$
y_{1}=B-Q(V \cdot B) \frac{1}{1+Q \cdot V}=\frac{B+V \times(B \times Q)}{1+Q \cdot V} .
$$

Next we consider the equation of the type

$$
y_{2}=B-A \times y_{2}
$$

which by iterating once leads to

$$
\left(1+A^{2}\right) y_{2}=B-A \times B+A\left(A \cdot y_{2}\right)
$$

which is again of the type A1) with $B \rightarrow(B-A \times$ $B) /\left(1+A^{2}\right), V \rightarrow-A$, and $Q \rightarrow A /\left(1+A^{2}\right)$ such that the solution reads

$$
y_{2}=\frac{B-A \times B+A(A \cdot B)}{1+A^{2}} .
$$

The combined type reads

$$
y_{3}+A \times y_{3}=B-Q\left(V \cdot y_{3}\right)
$$

where in a first step we consider the right hand side as a $B$ of the problem $(\mathrm{A} 3$ and get the solution according to A5). This leads to the problem A1) with $B \rightarrow(B-$ $A \times B+A(A \cdot B)) /\left(1+A^{2}\right)$ and $Q \rightarrow(Q-A \times Q+$ $A(A \cdot Q)) /\left(1+A^{2}\right)$ such that the solution can be written according to A2

$$
\begin{aligned}
y_{3} & =\frac{B-A \times B+A(A \cdot B)+V \times H}{1+A^{2}+Q \cdot V-V(A \times Q)+(A \cdot V)(A \cdot Q)} \\
H & =(B-A \times B+A(A \cdot B)) \times \frac{Q-A \times Q+A(A \cdot Q)}{1+A^{2}} \\
& =B \times Q+A \times(Q \times B)
\end{aligned}
$$

where the last equality is a matter of algebra. The final solution reads therefore

$$
\begin{aligned}
& y_{3}= \\
& \frac{B-A \times B+A(A \cdot B)+V \times[B \times Q+A \times(Q \times B)]}{1+A^{2}+Q \cdot V-V \cdot(A \times Q)+(A \cdot V)(A \cdot Q)} \\
& \equiv \frac{y_{3 z}}{y_{3 n}} .
\end{aligned}
$$

As a next complication we consider the vector equation where the scalar products appear with respect to two vectors

$$
y_{4}+A \times y_{4}+Q\left(V \cdot y_{4}\right)=B-P\left(T \cdot y_{4}\right)
$$

which is recast into the problem A6 with $B \rightarrow B-$ $P\left(T \cdot y_{4}\right)$ such that we obtain

$$
\begin{aligned}
& y_{4}=y_{3}-\left(T \cdot y_{4}\right) Q_{1} \\
& Q_{1}= \\
& \frac{P+P \times A+V \times(A \times(Q \times P)-Q \times P)+A(A \cdot P)}{y_{3 n}}
\end{aligned}
$$

which is the problem A1 with $V \rightarrow T, B \rightarrow y_{3}$ and $Q \rightarrow Q_{1}$ such that we obtain

$$
y_{4}=\frac{y_{3}-T \times\left(Q_{1} \times y_{3}\right)}{1+T \cdot Q_{1}} .
$$

The cross product in the numerator can be shown by somewhat lengthy calculation to be

$$
Q_{1} \times y_{3}=\frac{P \times B+A \times(B \times P)+V(B \cdot P \times Q)}{y_{3 n}}
$$

such that the final solution reads

$$
\begin{aligned}
& y_{4}= \\
& \frac{y_{3 z}-T \times[A \times(B \times P)-B \times P+V P \cdot(B \times Q)]}{y_{3 n}+T \cdot y_{4 h}} \\
& y_{4 h}= \\
& P+P \times A-V \times[A \times(P \times Q)+P \times Q]+A(A \cdot P)
\end{aligned}
$$

\section{Appendix B: Evaluation of operator forms}

In this appendix we evaluate the operator form

$$
\int_{0}^{\infty} d x \mathrm{e}^{i \bar{\omega} x} \mathrm{e}^{(\vec{b}+\vec{a}) \cdot \vec{\sigma} x}\left(S_{0}+\vec{\sigma} \cdot \vec{S}\right) \mathrm{e}^{(\vec{b}-\vec{a}) \cdot \vec{\sigma} x}
$$

with $\bar{\omega}=\omega+i / \tau$. First we rewrite the exponential of Pauli matrices to obtain

$$
\mathrm{e}^{(\vec{b} \pm \vec{a}) \cdot \vec{\sigma} x}=\cos c_{ \pm} x+i \vec{e}_{ \pm} \cdot \vec{\sigma} \sin c_{ \pm} x
$$

where we use

$$
c_{ \pm}=|b \pm a| ; \quad \vec{e}_{ \pm}=\frac{\vec{b} \pm \vec{a}}{|\vec{b} \pm \vec{a}|} .
$$

To evaluate the occurring products it is useful to deduce from $(\vec{a} \cdot \vec{\sigma})(\vec{b} \cdot \vec{\sigma})=\vec{a} \cdot \vec{b}+i \vec{\sigma} \cdot(\vec{a} \times \vec{b})$ the relations

$$
\vec{\sigma} \cdot(\vec{a} \cdot \vec{\sigma})=\vec{a}+i(\vec{a} \times \vec{\sigma}), \quad(\vec{a} \cdot \vec{\sigma}) \cdot \vec{\sigma}=\vec{a}-i(\vec{a} \times \vec{\sigma})(\mathrm{B} 4)
$$


with the help of which we find

$$
\begin{aligned}
& (\sigma \cdot \vec{a})(\vec{b} \times \vec{\sigma})=-((\sigma \cdot \vec{a}) \vec{\sigma} \times \vec{b})=-(\vec{a}-i(\vec{a} \times \vec{\sigma})) \times \vec{b} \\
& =-\vec{a} \times \vec{b}+i \vec{b} \times(\vec{\sigma} \times \vec{a})=-\vec{a} \times \vec{b}+i \vec{\sigma}(\vec{a} \cdot \vec{b})-i \vec{a}(\vec{b} \cdot \vec{\sigma}) .
\end{aligned}
$$

One obtains from $\mathrm{B} 2$

$$
\begin{aligned}
& \mathrm{e}^{(\vec{b}+\vec{a}) \cdot \vec{\sigma} x} \mathrm{e}^{(\vec{b}-\vec{a}) \cdot \vec{\sigma} x}=\cos c_{+} x \cos c_{-} x \\
& +i\left(\vec{e}_{+} \cdot \vec{\sigma}\right) \sin c_{+} x \cos c_{-} x+i\left(\vec{e}_{-} \cdot \vec{\sigma}\right) \sin c_{-} x \cos c_{+} x \\
& -\left[\vec{e}_{+} \cdot \vec{e}_{-}+i \vec{\sigma} \cdot\left(\vec{e}_{+} \times \vec{e}_{-}\right)\right] \sin c_{+} x \sin c_{-} x
\end{aligned}
$$

and

$$
\begin{aligned}
& \mathrm{e}^{(\vec{b}+\vec{a}) \cdot \vec{\sigma} x} \vec{\sigma} \mathrm{e}^{(\vec{b}-\vec{a}) \cdot \vec{\sigma} x}=\vec{\sigma} \cos c_{+} x \cos c_{-} x \\
& +i\left(\vec{e}_{+}-\vec{\sigma} \times \vec{e}_{+}\right) \sin c_{+} x \cos c_{-} x \\
& +i\left(\vec{e}_{-}+\vec{\sigma} \times \vec{e}_{+}\right) \sin c_{-} x \cos c_{+} x \\
& +\left[\vec{\sigma}\left(\vec{e}_{+} \cdot \vec{e}_{-}\right)-\left(\vec{\sigma} \cdot \vec{e}_{+}\right) \vec{e}_{-}-\left(\vec{\sigma} \cdot \vec{e}_{-}\right) \vec{e}_{+}+i\left(\vec{e}_{+} \times \vec{e}_{-}\right)\right] \\
& \quad \times \sin c_{+} x \sin c_{-} x .
\end{aligned}
$$

Now we evaluate the integrals over the cos and sin functions. Due to the positive imaginary part of $\omega$ the integral vanishes at the upper infinite limit and one has

$$
\begin{aligned}
& \int_{0}^{\infty} d x \mathrm{e}^{i \omega x} \cos (c x)=\frac{i \omega}{\omega^{2}-c^{2}} \\
& \int_{0}^{\infty} d x \mathrm{e}^{i \omega x} \sin (c x)=-\frac{c}{\omega^{2}-c^{2}} .
\end{aligned}
$$

We will need

$$
\left(c_{+} \pm c_{-}\right)^{2}=2\left(a^{2}+b^{2} \pm\left|a^{2}-b^{2}\right|\right) .
$$

which leads to either to $4 a^{2}$ or $4 b^{2}$ dependent whether $a^{2} \gtrless b^{2}$ and the \pm sign respectively. Therefore one obtains

$$
\begin{aligned}
& \int_{0}^{\infty} d x \mathrm{e}^{i \omega x} \cos \left(c_{+} x\right) \cos \left(c_{-} x\right)=\frac{i \omega}{2}\left(\frac{1}{\omega^{2}-4 b^{2}}+\frac{1}{\omega^{2}-4 a^{2}}\right) \\
& \int_{0}^{\infty} d x \mathrm{e}^{i \omega x} \frac{\sin \left(c_{ \pm} x\right) \sin \left(c_{\mp} x\right)}{\left|a^{2}-b^{2}\right|}=-\frac{2 i \omega}{\left(\omega^{2}-4 a^{2}\right)\left(\omega^{2}-4 b^{2}\right)} \\
& \int_{0}^{\infty} d x \mathrm{e}^{i \omega x}\left(\frac{\sin \left(c_{+} x\right) \cos \left(c_{-} x\right)}{|\vec{a}+\vec{b}|}+\frac{\sin \left(c_{-} x\right) \cos \left(c_{+} x\right)}{|\vec{a}-\vec{b}|}\right) \\
& =-\frac{2 \omega^{2}}{\left(\omega^{2}-4 a^{2}\right)\left(\omega^{2}-4 b^{2}\right)} \\
& \int_{0}^{\infty} d x \mathrm{e}^{i \omega x}\left(\frac{\sin \left(c_{+} x\right) \cos \left(c_{-} x\right)}{|\vec{a}+\vec{b}|}-\frac{\sin \left(c_{-} x\right) \cos \left(c_{+} x\right)}{|\vec{a}-\vec{b}|}\right) \\
& =\frac{8 \vec{a} \cdot \vec{b}}{\left(\omega^{2}-4 a^{2}\right)\left(\omega^{2}-4 b^{2}\right)} .
\end{aligned}
$$

This allows to calculate the different occurring integrals in (B1) with (B6) and (B7) as

$$
\begin{aligned}
& \int_{0}^{\infty} d x \mathrm{e}^{i \omega x} \mathrm{e}^{(\vec{b}+\vec{a}) \cdot \vec{\sigma} x} \mathrm{e}^{(\vec{b}-\vec{a}) \cdot \vec{\sigma} x} \\
& =\frac{4 \omega \vec{\sigma} \cdot(\vec{b} \times \vec{a})+i \omega\left(\omega^{2}-4 a^{2}\right)+8 i(\vec{\sigma} \cdot \vec{a})(\vec{a} \cdot \vec{b})-2 i \omega^{2}(\vec{\sigma} \cdot \vec{b})}{\left(\omega^{2}-4 a^{2}\right)\left(\omega^{2}-4 b^{2}\right)}
\end{aligned}
$$

and

$$
\begin{aligned}
& \int_{0}^{\infty} d x \mathrm{e}^{i \omega x} \mathrm{e}^{(\vec{b}+\vec{a}) \cdot \vec{\sigma} x} \vec{\sigma} \mathrm{e}^{(\vec{b}-\vec{a}) \cdot \vec{\sigma} x}=\{4 \omega(\vec{a} \times \vec{b})+8 i \vec{a}(\vec{a} \cdot \vec{b}) \\
& +i \omega\left[\vec{\sigma}\left(\omega^{2}-4 b^{2}\right)+4 \vec{b}(\vec{\sigma} \cdot \vec{b})-4 \vec{a}(\vec{\sigma} \cdot \vec{a})-2 \vec{b} \omega\right] \\
& \left.+8(\vec{a} \cdot \vec{b})(\vec{b} \times \vec{\sigma})+2 \omega^{2}(\vec{\sigma} \times \vec{a})\right\} \frac{1}{\left(\omega^{2}-4 a^{2}\right)\left(\omega^{2}-4 b^{2}\right)} .
\end{aligned}
$$

\section{Appendix C: Long wavelength expansion}

In order to discuss dispersion relations and collective modes we need the expansion of all polarization functions up to second order in wavelength appearing in terms of

$\Pi\left(\omega+\frac{i}{\tau}-\frac{\vec{p} \cdot \vec{q}}{m_{e}}\right)=\left(1-\frac{\vec{p} \cdot \vec{q}}{m_{e}} \partial_{\omega}+\frac{(\vec{p} \cdot \vec{q})^{2}}{m_{e}^{2}} \partial_{\omega}^{2}\right) \Pi\left(\omega_{+}\right)$

where we use $\omega_{+}=\omega+i / \tau$. A further wave-length term comes from the magnetic field dependence of the polarization function discussed in chapter IV.C which leads to a term linear in the magnetic field

$$
1-\frac{\omega_{c}}{2 m_{e}} \vec{q} \cdot\left(\vec{e}_{z} \times \vec{p}\right) \partial_{\omega}^{2}
$$

Any function of $\Sigma$ we can expand therefore as

$$
\Pi(\Sigma)=\left[1+\left(\frac{b_{\perp}^{2}}{2}+b_{3}\right) \Sigma_{n} \partial_{\Sigma_{n}}+\frac{b_{3}^{2}}{2} \Sigma_{n}^{2} \partial_{\Sigma_{n}}^{2}\right] \Pi\left(\Sigma_{n}\right)
$$

Summarizing we have to apply (C1), C2 and (C3) to all polarization function and calculate the momentum integration. Here we give the final results which may be obtained after some lengthy calculation. Since $b_{\perp}(\vec{p})$ is uneven and $b_{3}(p)$ is even in momentum, from various mean values with the momentum-even distributions only the following terms remain nonzero

$$
\begin{aligned}
\frac{\omega_{c}}{2 m_{e}} \sum_{p}\left(\begin{array}{c}
f \\
g
\end{array}\right) \vec{q} \cdot\left(\vec{e}_{z} \times \vec{p}\right) \vec{b}_{\perp}(p) & =\frac{\omega_{c}}{D} \vec{b}_{\perp}(q) \times \vec{e}_{z}\left(\begin{array}{c}
E_{f} \\
E_{g}
\end{array}\right) \\
\sum_{p}\left(\begin{array}{c}
f \\
g
\end{array}\right) \frac{(\vec{p} \cdot \vec{q})^{2}}{2 m_{e}^{2}} & =\frac{q^{2}}{D m_{e}}\left(\begin{array}{c}
E_{f} \\
E_{g}
\end{array}\right)
\end{aligned}
$$


where $D$ denotes the dimension and the mean (polarization) kinetic energy is denoted as

$$
\left(\begin{array}{l}
E_{f} \\
E_{g}
\end{array}\right)=\sum_{p}\left(\begin{array}{l}
f \\
g
\end{array}\right) \frac{p^{2}}{2 m_{e}}
$$

Here and in the following we use $\vec{q} \overrightarrow{\partial_{p}} b(p) \approx b(q)$ strictly valid only for linear spin-orbit coupling and neglect higher-order moments than $o\left(p^{2} b(p)\right)$. Besides (58) we will use further shorthand notations

$$
B_{g 3}=\sum_{p} b_{3} g, \quad B_{g i i}=\sum_{p} b_{\perp i}^{2} g, \quad \vec{B}_{\perp}=\vec{b}_{\perp}(q)
$$

and analogously for $g \leftrightarrow f$.

The equation for the density dipole-excitation is given by the first line of 46 and one needs the expansion of the polarizations

$$
\Pi_{0}=\frac{n q^{2}}{m_{e} \omega_{+}^{2}}+o\left(q^{3}\right)
$$

and analogously

$$
\vec{\Pi}=\frac{\vec{m} q^{2}}{m_{e} \omega_{+}^{2}}+o\left(q^{3}\right) .
$$

This means for neutral scattering the combinations $V_{0} \Pi_{0}$ and $V_{0} \vec{\Pi}$ vanish.

According to 47 we need the expansion of 51

$$
\begin{aligned}
& \vec{\Pi}_{g}=-i\left\{\vec { e } _ { z } \left[s_{0}-\frac{B_{g}^{2}}{2}\left(1-\Sigma_{n} \partial_{\Sigma_{n}}\right)+B_{g 3} \Sigma_{n} \partial_{\Sigma_{n}}\right.\right. \\
& \left.\left.+\frac{B_{g 33}}{2} \Sigma_{n}^{2} \partial_{\Sigma_{n}}^{2}+\frac{q^{2} E_{g}}{D m} \partial_{\omega}^{2}\right]-\vec{B}_{\perp} \times \vec{e}_{z} \frac{\omega_{c} E_{g}}{D} \partial_{\omega}^{2}\right\} \frac{2 \omega}{\omega^{2}-4 \Sigma_{n}^{2}}
\end{aligned}
$$

and also $+o\left(q^{3}\right)$

$$
\begin{aligned}
& \vec{\Pi}_{x f}=i\left\{\vec { e } _ { z } \left[\frac { q ^ { 2 } } { m } \left(n-\frac{B_{f}^{2}}{2}\left(1-\Sigma_{n} \partial_{\Sigma_{n}}\right)+B_{f 3} \Sigma_{n} \partial_{\Sigma_{n}}\right.\right.\right. \\
& \left.\left.+\frac{B_{f 33}}{2} \Sigma_{n}^{2} \partial_{\Sigma_{n}}^{2}\right) \partial_{\omega}-n B_{\perp 3} \Sigma_{n} \partial_{\Sigma_{n}}-B_{\perp 3} B_{f 3} \Sigma_{n}^{2} \partial_{\Sigma_{n}}^{2}\right] \\
& -\vec{B}_{\perp}\left(n-B_{f 3}\left(1-\Sigma_{n} \partial_{\Sigma_{n}}\right)\right) \\
& -\vec{B}_{\perp} \times \vec{e}_{z} B_{\perp 3} \frac{\omega_{c} E_{g}}{D}\left(1-\Sigma_{n} \partial_{\Sigma_{n}} \partial_{\omega}\right\} \frac{2 \Sigma_{n}}{4 \Sigma_{n}^{2}-\omega^{2}}
\end{aligned}
$$

such that we have the precession term $\vec{\Pi}_{2}=\vec{\Pi}_{x f}+\vec{\Pi}_{g}$.

For $\Pi_{3}$ we need besides (C8) according to 47)

$$
\begin{aligned}
& \vec{\Pi}_{x g}=i\left\{\vec{e}_{z} \times \vec{B}_{\perp}\left(s_{0}+B_{g 3} \Sigma_{n} \partial_{\Sigma_{n}}\right)\right. \\
& \left.+\left[\vec{B}_{\perp} B_{\perp 3}-\vec{e}_{z} B_{\perp}^{2}\right] \frac{\omega_{c} E_{g}}{D} \partial_{\omega}^{2}\right\} \frac{2 \Sigma}{4 \Sigma^{2}-\omega^{2}}
\end{aligned}
$$

and

$$
\begin{aligned}
& \vec{\Pi}_{e}=\left\{\vec{B}_{\perp}\left(s_{0}-B_{g 3}\left(1-\Sigma_{n} \partial_{\Sigma_{n}}\right)-B_{g 33} \Sigma_{n} \partial_{\Sigma_{n}}\right)\right. \\
& \left.+\vec{b}_{\perp}(q) \times \vec{e}_{z} \frac{\omega_{c} E_{g}}{D} \partial_{o} m e g a^{2}\right\} \frac{4 \Sigma_{n}^{2}}{\omega\left(4 \Sigma_{n}^{2}-\omega^{2}\right)}
\end{aligned}
$$

such that $\vec{\Pi}_{3}=o(q)$. The in-plane terms are a little bit more lengthy

$$
\begin{aligned}
& \overleftrightarrow{\Pi}_{x e}=2\left\{( \begin{array} { l l l } 
{ 1 } & { 0 } & { 0 } \\
{ 0 } & { 1 } & { 0 } \\
{ 0 } & { 0 } & { 0 }
\end{array} ) \left(s_{0}+\frac{q^{2} E_{g}}{m D} \partial_{\omega}^{2}+\left(B_{g 3}+\frac{B_{g}^{2}}{2}\right) \Sigma_{n} \partial_{\Sigma_{n}}\right.\right. \\
& \left.+\frac{B_{g 33}}{2} \Sigma_{n}^{2} \partial_{\Sigma_{n}}^{2}\right)+\left(\begin{array}{ccc}
0 & 0 & B_{\perp 2} \\
0 & 0 & -B_{\perp 1} \\
B_{\perp 2} & -B_{\perp 1} & 0
\end{array}\right) \frac{\omega_{c} E_{g}}{D} \partial_{\omega}^{2} \\
& \left.-\left(\begin{array}{ccc}
B_{g 11} & 0 & 0 \\
0 & B_{g 22} & 0 \\
0 & 0 & -B_{g}^{2}
\end{array}\right)\right\} \frac{2 \Sigma_{n}}{4 \Sigma_{n}^{2}-\omega_{+}^{2}}
\end{aligned}
$$

and

$$
\begin{aligned}
& \overleftrightarrow{\Pi}_{f e}=\left\{-B_{\perp 3}\left(\begin{array}{ccc}
1 & 0 & 0 \\
0 & 1 & 0 \\
0 & 0 & 0
\end{array}\right)\left(n \Sigma_{n} \partial_{\Sigma_{n}}+B_{f 3} \Sigma_{n}^{2} \partial_{\Sigma_{n}}^{2}\right)\right. \\
& +\left(\begin{array}{ccc}
2 B_{\perp 1} B_{\perp 2} & B_{\perp 2}^{2}-B_{\perp 1}^{2} & B_{\perp 2} \\
B_{\perp 2}^{2}-B_{\perp 1}^{2} & -2 B_{\perp 1} B_{\perp 2} & -B_{\perp 1} \\
B_{\perp 2} & -B_{\perp 1} & 0
\end{array}\right) \frac{\omega_{c} E_{f}}{D} \partial_{\omega}^{2} \\
& +\left[\left(\begin{array}{lll}
1 & 0 & 0 \\
0 & 1 & 0 \\
0 & 0 & 0
\end{array}\right)\left[\left(B_{f 3}+\frac{B_{f}^{2}}{2}\right) \Sigma_{n} \partial_{\Sigma_{n}}+\frac{B_{f 33}}{2} \Sigma_{n}^{2} \partial_{\Sigma_{n}}^{2}\right]\right. \\
& \left.\left.+\left(\begin{array}{ccc}
n-B_{f 11} & 0 & 0 \\
0 & n-B_{f 22} & 0 \\
0 & 0 & B_{f}^{2}
\end{array}\right)\right] \frac{q^{2}}{m} \partial_{\omega}\right\} \frac{4 \Sigma_{n}^{2}}{\omega\left(4 \Sigma_{n}^{2}-\omega_{+}^{2}\right)}
\end{aligned}
$$

The terms coming from the Mermin relaxation time become

$$
\begin{aligned}
\Pi_{0 \mu} & =-\frac{\partial_{\mu} n}{\omega_{+}}-\frac{n q^{2}}{m_{e} \omega_{+}^{3}}+o\left(q^{3}, b^{3}, \sigma_{n} b(q)^{2}\right) \\
\vec{\Pi}_{\mu} & =-\frac{\partial_{\mu} \vec{s}}{\omega_{+}}-\frac{s q^{2}}{m_{e} \omega_{+}^{3}} \vec{e}_{z}+o\left(q^{3}, b^{3}, \sigma_{n} b(q)^{2}\right)
\end{aligned}
$$

where we use 57 . The terms $\vec{\Pi}_{\mu e}$ and $\vec{\Pi}_{x \mu}$ vanish at this level of expansion.

\section{Long wave length expansion in quasi $2 \mathrm{D}$ systems}

In the cases discussed in this paper we are not interested in 3D spin-orbit coupling such that we can neglect the terms $b_{3}$. Summarizing the results of the last chapter we obtain for the coupled dispersion 46 the terms

$$
\Pi_{0}=\frac{n q^{2}}{m_{e} \omega_{+}^{2}}, \quad \vec{\Pi}=\frac{\vec{s} q^{2}}{m_{e} \omega_{+}^{2}}, \quad s=s_{0}-\frac{B_{g}^{2}}{2},
$$




$$
\begin{aligned}
& \vec{\Pi}_{2}=i \vec{e}_{z}\left\{\left[-s_{0}+\frac{B_{g}^{2}}{2}\left(1-\Sigma_{n} \partial_{\Sigma_{n}}\right)-\frac{q^{2} E_{g}}{D m_{e}} \partial_{\omega}^{2}\right] \frac{2 \omega}{\omega^{2}-4 \Sigma_{n}^{2}}\right. \text { together with (C15). } \\
& \left.-\vec{B}_{\perp} \times \vec{e}_{z} \frac{\omega_{c} E_{g}}{D} \partial_{\omega}^{2}+\frac{q^{2}}{m}\left(n-\frac{B_{f}^{2}}{2}\left(1-\Sigma_{n} \partial_{\Sigma_{n}}\right)\right) \frac{2 \Sigma_{n}}{4 \Sigma_{n}^{2}-\omega^{2}}\right\} \\
& +i \vec{B} \times \vec{e}_{z} \frac{\omega_{c} E_{g}}{D} \partial_{\omega}^{2} \frac{2 \omega}{\omega^{2}-4 \Sigma_{n}^{2}}-\vec{B}_{\perp} n \frac{2 \Sigma_{n}}{4 \Sigma_{n}^{2}-\omega^{2}}, \quad(\mathrm{C} 17) \\
& \vec{\Pi}_{3}=\frac{\vec{s} q^{2}}{m_{e} \omega_{+}^{2}}-i\left[\vec{e}_{z} \times \vec{B}_{\perp} s_{0}-\vec{e}_{z} B_{\perp}^{2} \frac{\omega_{c} E_{g}}{D} \partial_{\omega}^{2}\right] \frac{2 \Sigma}{4 \Sigma^{2}-\omega^{2}} \\
& +\vec{B}_{\perp} s_{0} \frac{4 \Sigma_{n}^{2}}{\omega\left(4 \Sigma_{n}^{2}-\omega^{2}\right)},
\end{aligned}
$$

and

$$
\begin{aligned}
& \overleftrightarrow{\Pi}=\left\{\left(\begin{array}{lll}
1 & 0 & 0 \\
0 & 1 & 0 \\
0 & 0 & 0
\end{array}\right)\left(s_{0}+\frac{q^{2} E_{g}}{m D} \partial_{\omega}^{2}+\frac{B_{g}^{2}}{2} \Sigma_{n} \partial_{\Sigma_{n}}\right)\right. \\
& \left.+\left\{\begin{array}{ccc}
-B_{g 11} & 0 & B_{\perp 2} \frac{\omega_{c} E_{g}}{D} \partial_{\omega}^{2} \\
0 & -B_{g 22} & -B_{\perp 1} \frac{\omega_{c} E_{g}}{D} \partial_{\omega}^{2} \\
B_{\perp 2} \frac{\omega_{c} E_{g}}{D} \partial_{\omega}^{2} & -B_{\perp 1} \frac{\omega_{c} E_{g}}{D} \partial_{\omega}^{2} & -B_{g}^{2}
\end{array}\right)\right\} \\
& \times \frac{4 \Sigma_{n}}{4 \Sigma_{n}^{2}-\omega_{+}^{2}} \\
& +\left\{\left(\begin{array}{ccc}
2 B_{\perp 1} B_{\perp 2} & B_{\perp 2}^{2}-B_{\perp 1}^{2} & B_{\perp 2} \\
B_{\perp 2}^{2}-B_{\perp 1}^{2} & -2 B_{\perp 1} B_{\perp 2} & -B_{\perp 1} \\
B_{\perp 2} & -B_{\perp 1} & 0
\end{array}\right) \frac{\omega_{c} E_{f}}{D} \partial_{\omega}^{2}\right. \\
& +\left(\begin{array}{ccc}
n+\frac{B_{f}^{2}}{2} \Sigma_{n} \partial_{\Sigma_{n}}-B_{f 11} & 0 & 0 \\
0 & n+\frac{B_{f}^{2}}{2} \Sigma_{n} \partial_{\Sigma_{n}}-B_{f 22} & 0 \\
0 & 0 & B_{f}^{2}
\end{array}\right) \\
& \left.\times \frac{q^{2}}{m_{e}} \partial_{\omega}\right\} \frac{4 \Sigma_{n}^{2}}{\omega\left(4 \Sigma_{n}^{2}-\omega_{+}^{2}\right)}
\end{aligned}
$$

1 N. M. R. Peres, F. Guinea, and A. H. Castro Neto, Phys. Rev. B 72, 174406 (2005).

2 I. J. Vera-Marun, B. J. van Wees, and R. Jansen, Phys. Rev. Lett. 112, 056602 (2014).

3 S. Das Sarma, S. Adam, E. H. Hwang, and E. Rossi, Rev. Mod. Phys. 83, 407 (2011).

${ }^{4}$ H. Tomita and J. Nakamura, J. Vac. Sci. Technol. B 31, 04D105 (2013).

5 E. P. Bashkin, C. da Providência, and J. da Providência, Phys. Rev. C 50, 2800 (1994).

${ }^{6}$ D. C. Langreth and J. W. Wilkins, Phys. Rev. B 6, 3189 (1972).

7 J. Sweer, D. C. Langreth, and J. W. Wilkins, Phys. Rev. B 13, 192 (1976).

8 A. J. Leggett and M. J. Rice, Phys. Rev. Lett. 20, 586 (1968), erratum Phys. Rev. Lett. 21506 (1968).

9 E. P. Bashkin, JETP Lett. 33, 8 (1981).

10 C. Lhuilier and F. Laloë, J. Phys. (Paris) 43, 197 (1982).

11 W. J. Gully and W. J. Mullin, Phys. Rev. Lett. 52, 1810
(1984).

12 E. P. Bashkin, Sov. Phys. Usp. 29, 238 (1986).

13 A. E. Ruckenstein and L. P. Lévy, Phys. Rev. B 39, 183 (1989).

14 B. R. Johnson, J. S. Denker, N. Bigelow, L. P. Lévy, J. H. Freed, and D. M. Lee, Phys. Rev. Lett. 52, 1508 (1984).

15 N. P. Bigelow, J. H. Freed, and D. M. Lee, Phys. Rev. Lett. 63, 1609 (1989).

16 W. J. Mullin and R. J. Ragan, J. Low Temp. Phys. 138, $73(2005)$.

17 H. J. Lewandowski, D. M. Harber, D. L. Whitaker, and E. A. Cornell, Phys. Rev. Lett. 88, 070403 (2002).

18 J. M. McGuirk, H. J. Lewandowski, D. M. Harber, T. Nikuni, J. E. Williams, and E. A. Cornell, Phys. Rev. Lett. 89, 090402 (2002).

19 J. E. Williams, T. Nikuni, and C. W. Clark, Phys. Rev. Lett. 88, 230405 (2002).

${ }^{20}$ W. J. Mullin and R. J. Ragan, Phys. Rev. A 74, 043607 (2006). 
21 T. Nikuni, J. E. Williams, and C. W. Clark, Phys. Rev. A 66, 043411 (2002).

22 S. Perisanu and G. Vermeulen, Phys. Rev. B 73, 214519 (2006).

23 P. J. Nacher, G. Tastevin, S. B. Crampton, and F. Laloë, J. Physique Lett. 45, L (1984).

24 E. P. Bashkin, Phys. Rev. B 44, 12440 (1991).

25 A. A. Burkov and L. Balents, Phys. Rev. B 69, 245312 (2004).

26 V. M. Edelstein, Phys. Rev. B 74, 193310 (2006).

27 V. Sih, W. H. Lau, R. C. Myers, A. C. Gossard, M. E. Flatté, and D. D. Awschalom, Phys. Rev. B 70, 161313 (2004).

28 R. A. Cover, G. Kalman, and P. Bakshi, Phys. Rev. D 20, 3015 (1979).

29 N. J. M. Horing and M. M. Yildiz, Phys. Rev. B 33, 3895 (1986).

30 V. López-Richard, G. E. Marques, and C. Trallero-Giner, J. Appl. Phys. 89, 6400 (2001).

31 O. Bleibaum, Phys. Rev. B 71, 235318 (2005).

32 K. Shizuya, Phys. Rev. B 75, 245417 (2007).

${ }^{33}$ K.-J. Lee, M. Stiles, H.-W. Lee, J.-H. Moon, K.-W. Kim, and S.-W. Lee, Phys. Rep. 531, 89 (2013).

34 A. Aperis, M. Georgiou, R. Roumpos, S. Tsonis, G. Varelogiannis, and P. B. Littlewood, Europhys. Lett. 83, 67008 (2008).

35 V. E. Demidov, H. Ulrichs, S. V. Gurevich, S. O. Demokritov, V. S. Tiberkevich, A. N. Slavin, A. Zholud, and S. Urazhdin, Nature comm. 5, 3179 (2014).

36 T. Kampfrath, M. Battiato, P. Maldonado, G. Eilers, J. Nötzold, S. Mährlein, V. Zbarsky, F. Freimuth, Y. Mokrousov, S. Blügel, et al., Nature Nanotechnology 8, 256 (2013).

37 R. Winkler, Spin-Orbit Coupling Effects in TwoDimensional Electron and Hole Systems (Springer - Verlag, Berlin Heidelberg, 2003).

${ }^{38}$ M. W. Wu, J. H. Jiang, and M. Q. Weng, Phys. Rep. 493, $61(2010)$.

${ }^{39}$ C. Ciccarelli, K. M. D. Hals, A. Irvine, V. Novak, Y. Tserkovnyak, H. Kurebayashi, A. Brataas, and A. Ferguson, Nature Nanotechnology 10, 50 (2015).

40 D. Fang, H. Kurebayashi, J. Wunderlich, K. Vỳbornỳ, L. P. Zârbo, R. P. Campion, A. Casiraghi, B. L. Gallagher, T. Jungwirth, and A. J. Ferguson, Nature Nanotechnology 6, 413 (2015).

41 R. H. Liu, W. L. Lim, and S. Urazhdin, Phys. Rev. Lett. 110, 147601 (2013).

42 V. M. Edelstein, Solid State Comm. 73, 233 (1990).

43 J. Borge, C. Gorini, G. Vignale, and R. Raimondi, Phys. Rev. B 89, 245443 (2014).

44 S. Wimmer, M. Seemann, K. Chadova, D. Ködderitzsch, and H. Ebert, Phys. Rev. B 92, 041101 (2015).

45 Y. V. Kobljanskyj, G. A. Melkov, A. A. Serga, A. N. Slavin, and B. Hillebrands (2015), arXiv:1503.04638.

46 D. H. Berman, M. Khodas, and M. E. Flatté, Phys. Rev. X 4, 011048 (2014).

47 L. B. Hu, J. Gao, and S. Q. Shen, Phys. Rev. B 68, 153303 (2003).

48 C. Grimaldi and P. Fulde, Phys. Rev. B 55, 15523 (1997).

49 K. Morawetz, Europhys. Lett. 67, 77 (2004).

50 T. Ando, J. Phys. Soc. Japan 75, 074716 (2006).

51 F. Guinea, Phys. Rev. B 75, 235433 (2007).

52 G. Gazzola, A. L. Cherchiglia, L. A. Cabral, M. C. Nemes, and M. Sampaio, Europ. Phys. Lett. 104, 27002 (2013).
53 V. Juričić, O. Vafek, and I. F. Herbut, Phys. Rev. B 82, 235402 (2010).

54 I. F. Herbut, V. Juričić, and O. Vafek, Phys. Rev. Lett. 100, 046403 (2008).

55 E. G. Mishchenko, Europ. Phys. Lett. 83, 17005 (2008).

${ }^{56}$ W. J. H. Leyland, G. H. John, R. T. Harley, M. M. Glazov, E. L. Ivchenko, D. A. Ritchie, I. Farrer, A. J. Shields, and M. Henini, Phys. Rev. B 75, 165309 (2007).

57 W. J. H. Leyland, R. T. Harley, M. Henini, A. J. Shields, I. Farrer, and D. A. Ritchie, Phys. Rev. B 76, 195305 (2007).

58 W. J. H. Leyland, R. T. Harley, M. Henini, A. J. Shields, I. Farrer, and D. A. Ritchie, Phys. Rev. B 77, 205321 (2008).

59 M. Pletyukhov and V. Gritsev, Phys. Rev. B 74, 045307 (2006).

60 S. M. Badalyan, A. Matos-Abiague, G. Vignale, and J. Fabian, Phys. Rev. B 79, 205305 (2009).

61 A. Scholz, T. Stauber, and J. Schliemann, Phys. Rev. B 86, 195424 (2012).

62 X.-F. Wang and T. Chakraborty, Phys. Rev. B 75, 033408 (2007).

63 G. Borghi, M. Polini, R. Asgari, and A. H. MacDonald, Phys. Rev. B 80, 241402 (2009).

64 A. Principi, M. Polini, and G. Vignale, Phys. Rev. B 80, 075418 (2009).

65 O. V. Gamayun, Phys. Rev. B 84, 085112 (2011).

66 S. Yuan, R. Roldán, and M. I. Katsnelson, Phys. Rev. B 84, 035439 (2011).

67 R. E. V. Profumo, R. Asgari, M. Polini, and A. H. MacDonald, Phys. Rev. B 85, 085443 (2012).

68 C. Triola and E. Rossi, Phys. Rev. B 86, 161408 (2012).

69 M. H. Schultz, A. P. Jauho, and T. G. Pedersen, Phys. Rev. B 84, 045428 (2011).

70 M. Busl, G. Platero, and A.-P. Jauho, Phys. Rev. B 85, 155449 (2012).

71 C. A. Ullrich and M. E. Flatté, Phys. Rev. B 68, 235310 (2003).

72 K. Morawetz, Phys. Rev. B 67, 115125 (2003).

73 R. Hakim, L. Mornas, P. Peter, and H. D. Sivak, Phys. Rev. D 46, 4603 (1992).

74 N. Mermin, Phys. Rev. B 1, 2362 (1970).

75 A. K. Das, J. Phys. F 5, 2035 (1975).

76 K. Morawetz, Phys. Rev. B 66, 075125 (2002), errata: Phys. Rev. B 88, 039905(E).

77 K. Morawetz, Phys. Rev. E 88, 022148 (2013).

78 G. Zala, B. N. Narozhny, and I. L. Aleiner, Phys. Rev. B 64, 214204 (2001).

79 V. P. Mineev, Phys. Rev. B 69, 144429 (2004).

80 A. J. Leggett, J. Phys. C 3, 448 (1970).

81 V. P. Mineev, Phys. Rev. B 72, 144418 (2005).

${ }^{82}$ R. Bala, R. Moudgil, S. Srivastava, and K. Pathak, European Phys. J. B 87, 5 (2014).

83 S. DasSarma, Solid State Commun. 36, 357 (1980).

84 W. Nicolazzi and S. Pillet, Phys. Rev. B 85, 094101 (2012).

85 A. L. Chernyshev, A. H. Castro Neto, and A. R. Bishop, Phys. Rev. Lett. 84, 4922 (2000).

86 P. Schwab, M. Dzierzawa, C. Gorini, and R. Raimondi, Phys. Rev. B 74, 155316 (2006).

87 M. A. Brand, A. Malinowski, O. Z. Karimov, P. A. Marsden, R. T. Harley, A. J. Shields, D. Sanvitto, D. A. Ritchie, and M. Y. Simmons, Phys. Rev. Lett. 89, 236601 (2002).

88 X. Du, L. Luo, B. Clancy, and J. E. Thomas, Phys. Rev. Lett. 101, 150401 (2008). 
89 U. Ebling, A. Eckardt, and M. Lewenstein, Phys. Rev. A 84, 063607 (2011).

90 A. Malinowski, R. S. Britton, T. Grevatt, R. T. Harley, D. A. Ritchie, and M. Y. Simmons, Phys. Rev. B 62, 13034 (2000).

91 P. H. Song and K. W. Kim, Phys. Rev. B 66, 035207 (2002).

92 M. Q. Weng and M. W. Wu, Phys. Rev. B 68, 075312 (2003).

93 I. B. Bernstein, Phys. Rev. 109, 10 (1958).

${ }^{94}$ M. Walter, G. Zwicknagel, and C. Toepffer, Eur. Phys. J.
D 35, $527(2005)$.

95 Morawetz, K., EPL 104, 27005 (2013).

96 P. Vasilopoulos, Phys. Rev. B 32, 771 (1985).

97 C. M. V. Vliet and P. Vasilopoulos, Journal of Physics and Chemistry of Solids 49, 639 (1988).

98 There is a puzzling difference in the denominator. We have instead of the square of scalar product $(\vec{c} \cdot \vec{z})^{2}$ now $c^{2} z^{2}$ which difference is just the squared cross product. Since we restrict later to linear orders in $q \partial_{p}$, i.e. linear orders in $\vec{c}$ we can neglect this difference. 